\title{
Análise dos golos no Mundial de Futebol da Rússia 2018
}

\section{Análisis de goles en la Copa del Mundo de Rusia 2018}

\section{Goals Analysis in the Russian World Cup 2018}

\author{
Santos, $F^{1,2,4}$, Belchior, $D^{3}$, Rodrigues, $M^{2,3}$, Sousa, $P^{3,4}$, Pinheiro, V ${ }^{3,4}$ \\ ${ }^{1}$ Escola Superior de Educação - Instituto Politécnico de Setúbal, Setúbal, Portgal, ${ }^{2}$ Faculdade de \\ Motricidade Humana - Universidade de Lisboa, Cruz Quebrada, Portugal; ${ }^{3}$ Instituto Superior de Lisboa \\ e Vale do Tejo, Odivelas, Portugal, ${ }^{4}$ Centro de Investigação em Qualidade de Vida - Instituto \\ Politécnico de Leiria, Leiria, Portugal
}

\begin{abstract}
RESUMO
O golo é um indicador crucial da análise de desempenho no futebol. O objetivo do nosso estudo foi analisar o golo e fases anteriores desde a recuperação da bola. Foram analisados um total de 169 golos da fase de grupos e eliminação do Mundial de 2018. As observações foram feitas através do programa LINCE e os dados foram analisados utilizando o programa HOISAN e SPSS Statistics 24. Os dados foram analisados através de estatística descritiva, gráfica, teste exato de Fisher, U-Mann Whitney e coordenadas polares. Foi verificado que a recuperação da bola acontece preferencialmente por falta do adversário, nas zonas de meio-campo ofensivo. O último passe é registado com muita frequência nas zonas ofensivas, em resultado do ataque posicional, com o pé-direito e dentro da grande área. Foi ainda verificado a importância dos esquemas táticos na obtenção do golo. As coordenadas polares mostram relações de ativação entre a finalização e os outros comportamentos técnico-táticos, reforçando e complementando a análise notacional. Concluímos que a análise notacional, complementada com tratamento estatístico e análise de coordenadas polares revelam informações relevantes para analisar o desempenho em competição e planear o processo de treino. Palavras chave: Observação e análise, análise do desempenho em competição, análise notational, coordenadas polares, golo no futebol
\end{abstract}

\section{RESUMEN}

El gol es un indicador crucial del análisis del rendimiento del fútbol. El objetivo de nuestro estudio fue analizar el gol y etapas anteriores desde la recuperación del balón. Se analizaron un total de 169 goles en la fase de grupos y eliminación del Mundial 2018. Las observaciones se realizaron con el programa LINCE y los datos se analizaron con el programa HOISAN y SPSS Statistics 24. Los datos se analizaron mediante estadísticas descriptivas y gráfica, prueba exacta de Fisher, U-Mann Whitney y coordenadas polares. Se verificó que la recuperación del balón ocurre preferentemente por falta del oponente, en las zonas del mediocampo ofensivo. El último pase se registra muy a menudo en las zonas ofensivas, como consecuencia del ataque posicional, con el pie derecho y dentro del área de penalti. También se comprobó la importancia de los esquemas tácticos para obtener el gol. Las coordenadas polares muestran las relaciones de activación entre la finalización y los otros comportamientos técnico-tácticos, reforzando y complementando el análisis notacional. Concluimos que el análisis notacional, complementado con el tratamiento estadístico y análisis de coordenadas polares, revela información relevante para analizar el desempeño en competición y planificar el proceso de entrenamiento.

Palabras clave: Observación y análisis, análisis de rendimiento de competición, análisis notacional, coordenadas polares, gol de fútbol. 


\section{Santos et al.}

\section{ABSTRACT}

The goal is a crucial indicator of performance analysis in football. The aim of our study was to analyze the goal and previous phases since the recovery of the ball. 169 goals from group stage and elimination of the 2018 World Cup were analyzed. The observations were made through the LINCE program and the data were analyzed using the HOISAN and SPSS Statistics 24 program. The data were analyzed using descriptive, graphic statistics, Fisher's exact test, U-Mann Whitney and polar coordinates. It was verified that the recovery of the ball happens preferably due to fault of the opponent, in the areas of offensive midfield. The last pass is recorded very often in the offensive zones, as a result of the positional attack, with the right foot and inside the large area. It was also verified the importance of tactical schemes in obtaining the goal. The polar coordinates show activation relationships between the finalization and the other technical-tactical behaviors, reinforcing and complementing the notational analysis. We conclude that the notational analysis, complemented with statistical analysis and polar coordinate analysis, reveal relevant information to analyze performance in competition and plan the training process.

Keywords: Observation and analysis, competition performance analysis, notational analysis, polar coordinates, soccer goal.

\section{INTRODUÇÃO}

No futebol profissional, como é o caso das seleções nacionais num campeonato do mundo, a análise do desempenho é de grande importância. O objetivo da análise de desempenho é recolher informações sobre o desempenho desportivo dos jogadores e da equipa, a fim de ajudar os treinadores na sua tomada de decisão (O'Donoghue, 2006). A recolha de informação proveniente da observação e análise do jogo torna-se também fundamental para o processo de treino, uma vez que é espaço que os treinadores têm para melhorar os jogadores e equipa, preparando-os para a dinâmica e complexidade da competição (Sampaio \& Maçãs, 2012; Tassi et al., 2018; Vives-Ribó \& Rabassa, 2020).

Diversas formas de observação e análise têm sido aplicadas por investigadores e analistas de desempenho (análise centróide e de dispersão de uma equipa, análise das redes, análise sequencial, deteção de T-patterns) para uma melhor compreensão dos comportamentos táticos (Sarmento et al., 2018). A análise notacional é outras das formas que nos permite fazer uma análise dos diferentes aspetos do desempenho (técnico, tático), através do registo de indicadores e da elaboração de uma base de dados estatística (Hughes \& James, 2008), podendo ser complementada com o recurso a técnicas estatísticas para uma melhor compreensão do desempenho dos jogadores e equipas (Liu et al., 2016; Nevill et al., 2002). Outra forma que pode contribuir para os objetivos referidos da análise do desempenho, é o recurso às coordenadas polares. As coordenadas polares permitem realizar análise de desempenho
(Anguera \& Hernández-Mendo, 2015) no plano individual (Amatria et al., 2019a; Castañer et al., 2016, 2017; Maneiro et al., 2018) e no plano coletivo (DíazDíaz et al., 2018; Maneiro et al., 2018), através da identificação de relações de excitação ou inibição do comportamento focal (comportamento condicionante) com os restantes comportamentos do sistema de observação (Amatria et al., 2019a; Castellano \& Hernández-Mendo, 2003).

O golo é um indicador crucial do desempenho individual e coletivo (Amatria et al., 2019b) bem como do sucesso tático de uma equipa de futebol (Joaquin Gonzalez-Rodenas et al., 2020), pelo que é importante compreender quais os fatores que contribuem para a criação eficaz de situações de golo (GonzálezRódenas et al., 2019).

A forma como a equipa recupera a bola influencia a transição subsequente da defesa-ataque e a fase ofensiva. Barreira et al. (2013) constatou que a recuperação da bola por tackle seguido de passe promove a transição para as zonas de finalização, a interceção tende a induzir o ataque posicional e é através da ação do guarda-redes que as equipas procuram mudar o centro do jogo para zonas de menor pressão do adversário. As ações técnico-táticas individuais defensivas de interceção são promotoras da criação de situações de finalização e da obtenção do golo (Barreira et al., 2013; Espada et al., 2018; Hughes \& Lovell, 2019; Liu et al., 2016). As zonas em que a bola é recuperada, tem se revelado também relevantes para a concretização de golos (Jones et al., 2004). Estudos mostraram que as recuperações de bola em zonas próximas da baliza adversária são mais vantajosas para finalizar com eficácia (Hughes \& 


\section{Golos no Campeonato do Mundo na Rússia}

Lovell, 2019; Tenga et al., 2010). No entanto, existem estudos que demonstram também a eficácia no processo ofensivo em resultado da recuperação de bola na zona central do meio-campo defensivo (Barreira et al., 2013; Espada et al., 2018). As zonas de passe final para finalização ocorrem preferencialmente nas zonas do meio-campo ofensivo e zonas ofensivas (último terço do campo) (Amatria et al., 2019b; Espada et al., 2018; González-Ródenas et al., 2019). Os esquemas táticos (situações de bola parada) revelam-se importantes para a concretização do golo (González-Ródenas et al., 2019; Joaquín Gonzalez-Rodenas et al., 2017), bem como o método de jogo ataque rápido e contra-ataque (Espada et al., 2018; Joaquín Gonzalez-Rodenas et al., 2017; Liu et al., 2015; Tenga et al., 2010). Os estudos efetuados têm revelado ainda que as finalizações em golo são registadas com mais frequência dentro da área de grande penalidade (Acar et al., 2009; Amatria et al., 2019b; Espada et al., 2018). Quanto à forma de finalizar, os estudos verificaram um maior número de golos obtidos com o pé direito, pé esquerdo e cabeça (Acar et al., 2009; Espada et al., 2018).

Tendo em conta o referido, pretendemos verificar como as seleções de alto rendimento de futebol recuperam a bola e construem as situações de finalização recorrendo à análise notacional e procurando verificar de que forma a fase da competição influencia as variáveis em estudo. É também objetivo do nosso estudo, recorrendo à técnica de coordenadas polares, verificar relações de excitação e inibição entre a conduta focal (finalização) e as restantes comportamentos técnico-táticos (recuperação da bola, último passe, método para obtenção do golo e forma de finalização).

\section{MATERIAIS E MÉTODOS}

O nosso estudo foi realizado tendo por base a metodologia observacional, tipo de investigação científica, que permite analisar comportamentos de jogadores e equipa no jogo de futebol (Castellano \& Hernández-Mendo, 2003; Chacón-Moscoso et al., 2018). Esta característica confere ao estudo uma validade ecológica, uma vez que a análise dos comportamentos é feita em contexto natural, não os dissociando das influências externas (Portell, Anguera, Hernández-Mendo, et al., 2015) e de uma forma não intrusiva permite medir as relações de interação entre os diferentes jogadores (Maneiro et al.,
2018), recorrendo a instrumento de observação elaborado especificamente (Anguera \& HernándezMendo, 2014). O desenho observacional do estudo é ideográfico, seguimento e multidimensional (I/P/M) (Anguera et al., 2018). Ideográfico, porque analisa os vários elementos que constituem uma equipa de futebol, num momento competitivo (pontual) e multidimensional uma vez que os comportamentos analisados têm vários níveis de resposta.

\section{Amostra}

A amostra observacional é constituída pelos golos marcados pelas 32 seleções participantes no Mundial da Rússia $2018 \quad(n=169)$. Os golos obtidos são relativos às concretizações registadas durante o tempo regulamentar, sendo que as grandes penalidades para desempate do jogo não foram consideradas. A análise da sequência desde o momento de recuperação da posse da bola até à finalização foi garantida para toda a amostra.

\section{Instrumentos}

O instrumento observacional (Tabela 1) utilizado para codificar as ações, desde a recuperação de bola até à finalização, foi o Sistema de Observação do Golo no Futebol (SOGF). Este instrumento foi desenvolvido e validado por Santos et al. (2016), tendo já sido utilizado em outras investigações (Espada et al., 2018; Santos et al., 2017).

A figura 1 e 2 ilustram a divisão do campo e das zonas de finalização.

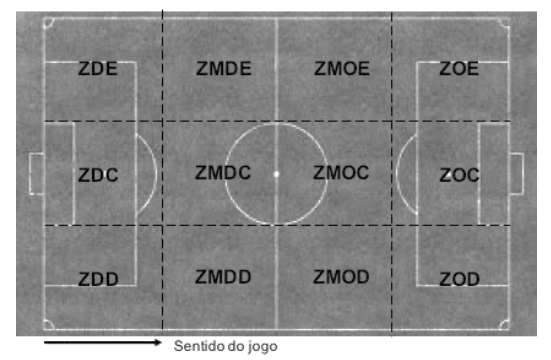

Figura 1. Campograma de divisão em zonas do terreno de jogo (adaptado de Gréhaigne, Mahut e Fernandez, 



\section{Golos no Campeonato do Mundo na Rússia}

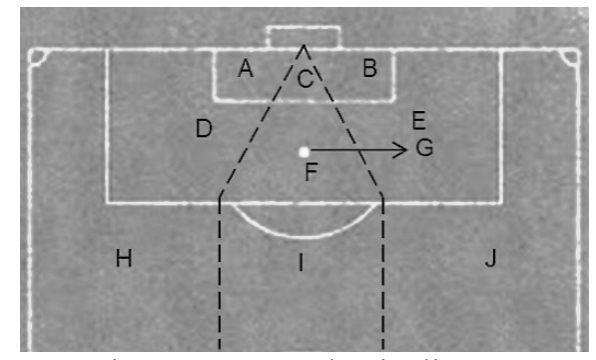

Figura 2. Zonas de Finalização

Tabela 1. Sistema de observação do golo no futebol (SOGF)

\begin{tabular}{|c|c|c|c|}
\hline Critério & Categorias & Código & \\
\hline \multirow{10}{*}{$\begin{array}{l}\text { Formas de } \\
\text { recuperação da } \\
\text { bola }\end{array}$} & Guarda-Redes & GR & $\begin{array}{l}\text { Ação do guarda-redes que permite a recuperação da bola com as mãos ou } \\
\text { com os pés resultante de um passe longo, cruzamento, remate ou esquema } \\
\text { tático. }\end{array}$ \\
\hline & Interceção & INT & $\begin{array}{l}\text { Ação em que a equipa recupera a posse de bola, quando esta é proveniente } \\
\text { de um remate ou de um passe entre dois adversários. }\end{array}$ \\
\hline & Duelo aéreo & DA & Ação de recuperação da bola através da disputa de bola no espaço aéreo \\
\hline & Desarme & DES & $\begin{array}{l}\text { Ação que procura interferir sobre a bola, recuperando-a, no confronto direto } \\
\text { com um adversário, normalmente em situações de } 1 \times 1 \text {. }\end{array}$ \\
\hline & Bola Perdida & BP & $\begin{array}{c}\text { A recuperação resulta de uma ação executada pelo adversário em que o } \\
\text { principal objetivo foi tirar a bola de um espaço vital ou de uma zona de } \\
\text { pressão. }\end{array}$ \\
\hline & Falta & FALTA & $\begin{array}{l}\text { A recuperação da bola é em resultado de uma falta realizada pelo } \\
\text { adversário, sendo assinalada livre direto, indireto ou grande penalidade. }\end{array}$ \\
\hline & $\begin{array}{l}\text { Saída de bola pelas linhas finais do } \\
\text { campo }\end{array}$ & SBLF & $\begin{array}{l}\text { A recuperação da bola é feita quando esta ultrapassa a linha final, tendo } \\
\text { sido tocada em último lugar pelo adversário, dando lugar à marcação de } \\
\text { pontapé de baliza ou de pontapé de canto. }\end{array}$ \\
\hline & $\begin{array}{l}\text { Saída de bola pelas linhas laterais do } \\
\text { campo }\end{array}$ & SBLL & $\begin{array}{l}\text { A recuperação da bola é feita quando ultrapassa uma das linhas laterais, } \\
\text { tendo sido tocada em último lugar pelo adversário, dando lugar à marcação } \\
\text { de lançamento de linha lateral. }\end{array}$ \\
\hline & Saída de bola no meio-campo & SBMC & $\begin{array}{l}\text { A recuperação de bola é feita no início das partes do jogo }\left(1^{\circ} \text { e } 2^{\circ} \text { parte }\right) \text { e } \\
\text { após um golo sofrido. }\end{array}$ \\
\hline & Comportamento Fair-Play & CFP & $\begin{array}{l}\text { A recuperação da bola é resultado de um comportamento de fair-play, tendo } \\
\text { este dado a bola. }\end{array}$ \\
\hline \multirow{14}{*}{$\begin{array}{l}\text { Zona de } \\
\text { recuperação da } \\
\text { bola }\end{array}$} & Zona Defensiva Esquerda & ZRDE & \multirow{14}{*}{ Figura 1} \\
\hline & Zona Defensiva Central & ZRDC & \\
\hline & Zona Defensiva Direita & ZRDD & \\
\hline & Zona Média Defensiva Esquerda & ZRMDE & \\
\hline & Zona Média Defensiva Central & ZRMDC & \\
\hline & Zona Média Defensiva Direita & ZRMDD & \\
\hline & Zona Média Ofensiva Esquerda & ZRMOE & \\
\hline & Zona Média Ofensiva Central & ZRMOC & \\
\hline & Zona Média Ofensiva Direita & ZRMOD & \\
\hline & Zona Ofensiva Esquerda & ZROE & \\
\hline & Zona Ofensiva Central & ZROC & \\
\hline & Zona Ofensiva Direita & ZROD & \\
\hline & Linha Lateral & LL & \\
\hline & Linha Final & LF & \\
\hline \multirow{13}{*}{$\begin{array}{l}\text { Zona do último } \\
\text { passe para } \\
\text { finalização }\end{array}$} & Zona Defensiva Esquerda & ZDE & \multirow{12}{*}{ Figura 1} \\
\hline & Zona Defensiva Central & ZDC & \\
\hline & Zona Defensiva Direita & ZDD & \\
\hline & Zona Média Defensiva Esquerda & ZMDE & \\
\hline & Zona Média Defensiva Central & ZMDC & \\
\hline & Zona Média Defensiva Direita & ZMDD & \\
\hline & Zona Média Ofensiva Esquerda & ZMOE & \\
\hline & Zona Média Ofensiva Central & ZMOC & \\
\hline & Zona Média Ofensiva Direita & ZMOD & \\
\hline & Zona Ofensiva Esquerda & ZOE & \\
\hline & Zona Ofensiva Central & $\mathrm{ZOC}$ & \\
\hline & Zona Ofensiva Direita & ZOD & \\
\hline & Sem passe/Finalização & SPF & $\begin{array}{l}\text { Situações em que a recuperação da bola, resulta também finalização (ex.: } \\
\text { defesa do guarda-redes seguido de finalização; jogador executa o passe para } \\
\text { o adversário que finaliza) }\end{array}$ \\
\hline
\end{tabular}




\section{Santos et al.}

\begin{tabular}{|c|c|c|c|}
\hline \multirow{11}{*}{$\begin{array}{c}\text { Método para } \\
\text { obtenção do golo }\end{array}$} & Contra-Ataque & CA & $\begin{array}{l}\text { Rápida transição defesa-ataque, com um reduzido tempo de construção } \\
(<10 \text { '’), pouco jogadores envolvidos, poucas ações técnico-táticas, elevado } \\
\text { ritmo de decisão, com a equipa adversária desorganizada. }\end{array}$ \\
\hline & Ataque Rápido & AR & $\begin{array}{c}\text { Rápida transição defesa-ataque, com um reduzido tempo de construção, } \\
\text { elevado ritmo de decisão, apesar da equipa adversária estar organizada } \\
\text { defensivamente. }\end{array}$ \\
\hline & Ataque Posicional & AP & $\begin{array}{l}\text { Maior tempo para construção do processo ofensivo, procurando maior } \\
\text { segurança na construção de situações de finalização. } \\
\text { Serão codificadas nesta categoria todas as situações em não haja claramente } \\
\text { transição rápida defesa-ataque. }\end{array}$ \\
\hline & Penalti & PEN & Golos resultantes da marcação de uma grande penalidade \\
\hline & Livre Direto & LD & Golos resultantes da marcação de livres diretos - remate direto à baliza \\
\hline & Livre Indireto & LI & $\begin{array}{l}\text { Golos resultantes da marcação de livres indiretos ( } 2 \text { ou mais toques). São } \\
\text { resultado de cruzamentos ou pontapés diretos para a zona da área. }\end{array}$ \\
\hline & Lançamento Linha Lateral & LLL & Golos resultantes de lançamentos de linha lateral. \\
\hline & Canto Longo & $\mathrm{CL}$ & Golos resultantes da marcação de pontapés de canto. \\
\hline & Canto Curto & $\mathrm{CC}$ & $\begin{array}{c}\text { Golos resultantes da marcação de pontapés de canto que envolvem } \\
\text { combinações até } 4 \text { passes. }\end{array}$ \\
\hline & Pontapé de baliza & PB & $\begin{array}{l}\text { Golos resultantes da marcação direta do pontapé de baliza. } \\
\text { Golos na sequência do começo e recomeço do jogo no 1/2 campo. }\end{array}$ \\
\hline & Bola de saída & BS & $\begin{array}{l}\text { Situações de golo resultantes de uma bola de saída, em que existe até } 4 \\
\text { passes, serão codificadas nesta categoria. }\end{array}$ \\
\hline \multirow{6}{*}{$\begin{array}{l}\text { Forma de } \\
\text { obtenção da } \\
\text { finalização }\end{array}$} & Pé Direito & PD & \multirow{6}{*}{$\begin{array}{c}\text { A finalização para golo é realizada pelo jogador com o pé direito. } \\
\text { A finalização para golo é realizada pelo jogador com o pé esquerdo. } \\
\text { A finalização para golo é realizada pelo jogador com a cabeça. } \\
\text { A finalização para golo é realizada pelo jogador com o peito. } \\
\text { A finalização para golo é realizada pelo jogador com outra parte do corpo. } \\
\text { A finalização para golo é conseguida através de um toque na bola de um } \\
\text { jogador da equipa adversária. }\end{array}$} \\
\hline & Pé Esquerdo & $\mathrm{PE}$ & \\
\hline & Cabeça & CAB & \\
\hline & Peito & $\mathrm{P}$ & \\
\hline & Outro & OUT & \\
\hline & Autogolo & $\mathrm{AG}$ & \\
\hline \multirow{10}{*}{$\begin{array}{l}\text { Zona de } \\
\text { Finalização }\end{array}$} & Zona A & $\mathrm{A}$ & \multirow{10}{*}{ Figura 2} \\
\hline & Zona B & B & \\
\hline & Zona C & $\mathrm{C}$ & \\
\hline & Zona D & D & \\
\hline & Zona E & $\mathrm{E}$ & \\
\hline & Zona $\mathrm{F}$ & $\mathrm{F}$ & \\
\hline & Zona $\mathrm{G}$ & G & \\
\hline & Zona $\mathrm{H}$ & $\mathrm{H}$ & \\
\hline & Zona I & I & \\
\hline & Zona $\mathrm{J}$ & $\mathrm{J}$ & \\
\hline
\end{tabular}

\section{Fiabilidade}

A fiabilidade é uma das questões-chave na codificação de comportamentos através de instrumentos observacionais de categorias (Moreno Cuerva \& Goméz Ruano, 2017; Losada \& Arnau, 2000) (Tabela 2). Assim, e para que os dados resultantes da observação tenham qualidade (Portell, Anguera, \& Chacón-Moscoso, 2015) os observadores foram treinados (Brewer \& Jones, 2002) e, em seguida, a fiabilidade intra-observadora e inter-observador foi testada para todos os critérios (Blanco-Villaseñor et al., 2014), utilizando a medida de concordância Kappa de Cohen (Cohen, 1960). Os valores de fiabilidade inter e intra-observador $(k>0,90)$ demonstram boa consistência, estabilidade e concordância da observação.

Tabela 2. Resultados da fiabilidade inter e intra observador

\begin{tabular}{cccc}
\hline Critério & Fiabilidade Intra & Fiabilidade Intra & Fiabilidade Inter \\
Observador 1 $(k)$ & Observador 2 $(k)$ & Observadores $(k)$ \\
\hline Formas de recuperação da bola & 1.0 & 0.92 & 0.95 \\
Zona de recuperação da bola & 1.0 & 0.93 & 0.91 \\
Zona do último passe para finalização & 0.92 & 1.0 & 0.90 \\
Método para obtenção do golo & 1.0 & 0.92 & 0.90 \\
Forma de obtenção da finalização & 1.0 & 1.0 & 1.0 \\
Zona de Finalização & 0.91 & 1.0 & 0.90 \\
Média $(M)$ & 0.97 & 0.96 & 0.92 \\
\hline
\end{tabular}





\section{Santos et al.}

\section{Procedimentos}

Os vídeos dos golos foram obtidos através da plataforma InStat ${ }^{\circledR}$ e foram posteriormente observados e codificados utilizando o software de computador Lince $^{\circledR}$ (Gabin et al., 2012).

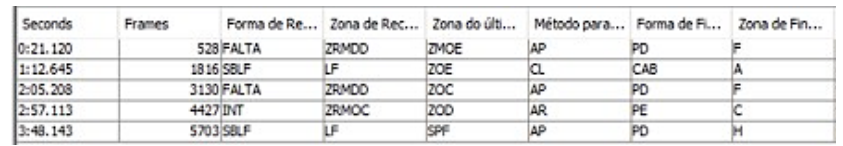

Figura 3. Exemplo da codificação dos golos no programa Lince ${ }^{\circledR}$

O programa Lince $^{\circledR}$ permite a exportação dos dados para o Microsoft Excel ${ }^{\mathbb{B}}$, que foi posteriormente inserido no Software SPSS Statistics $24^{\circledR}$. O mesmo programa exporta também os ficheiros para análise sequencial multievent no programa SDIS-GSEQ ${ }^{\circledR}$ (Bakeman \& Quera, 2011) e posterior análise das coordenadas polares através do programa $H_{O I S A N}{ }^{\mathbb{B}}$ (Hernández-Mendo et al., 2014)

\section{Análise de dados}

A análise de dados foi realizada utilizando o programa de computador SPSS Statistics $24^{\circledR}$. Foi realizada uma análise descritiva (soma, desvio padrão e média $-M \pm$ $D P)$ e a estatística gráfica recorrendo ao gráfico de barras de erros para um intervalo de confiança de $95 \%$. A normalidade da distribuição foi testada através dos testes Kolmogorov-Smirnov e Shapiro-Wilk, tendo-se verificado que não existe normalidade na distribuição.

A comparação dos golos obtidos tendo em conta a fase da competição, a fim de verificar os fatores potencialmente associados à obtenção do golo, foi realizado através do teste exato de Fisher $(p<0,05)$, com o cálculo correspondente do coeficiente de contingência.

A comparação entre os golos marcados na fase de grupos e a fase a eliminar foi feita através do teste $U$ Mann-Whitney $(p<0,05)$.

Para detetar relações de excitação e inibição entre a conduta focal (golo obtido na zona de finalização) e os comportamentos de recuperação da bola, último passe, método para obtenção do golo e forma de finalização utilizamos a técnica de coordenadas polares (Amatria et al., 2019a , Jiménez-Salas et al, 2020). A técnica de análise das coordenadas polares tem por base $Z_{\text {sum }}$ de
Cochran (1954), tendo por base o princípio de que a soma de um número $N$ e pontuações $z$ independentes se distribuem normalmente, $\operatorname{com} Z=0$ e $s=N$ (Maneiro et al., 2018).

$$
\begin{gathered}
Z_{\text {sum }=\sum_{l}^{m} \frac{Z}{\sqrt{n}}} \\
(n-\text { número de retardos })
\end{gathered}
$$

Análise é feita prospectivamente $\left(\begin{array}{lll}+1 & \mathrm{a} & +5\end{array}\right) \mathrm{e}$ retrospetivamente (-1 a -5$)$, sendo obtido um vetor por cada conduta relacionada com a conduta focal, com um determinado ângulo e raio, que se pode localizar em quatro quadrantes (Amatria et al., 2019a). Os vetores são considerados significativos $(p<0,05)$, quando apresentam um cumprimento vetorial de radio >1,96 (Díaz-Díaz et al., 2018).

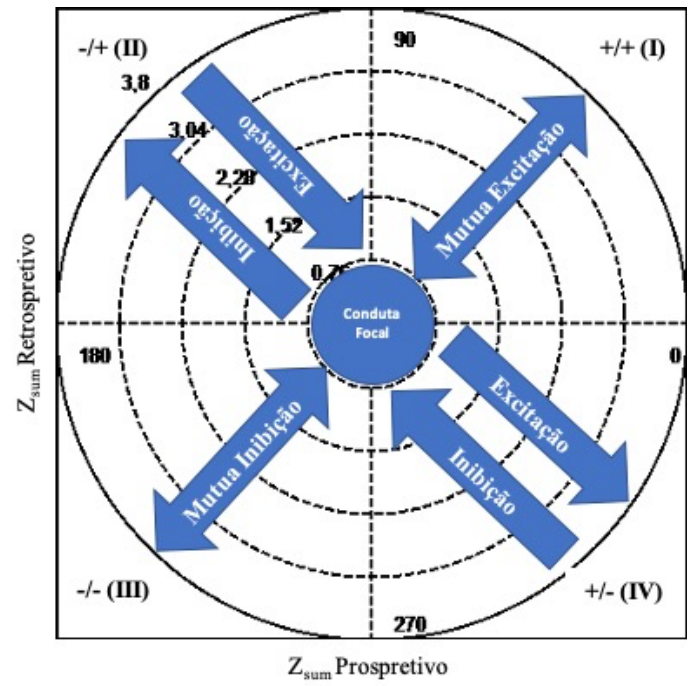

Figura 4. Representação gráfica das relações de excitação e inibição entre a conduta focal e as condutas condicionais, tendo em conta os quadrantes que os vetores estão localizados

A representação gráfica das coordenadas polares foi realizado através da Shyny App (Rodríguez-Medina et al, 2019). O tratamento dos dados realizado está de acordo com o sugerido para o desenho observacional do nosso estudo (Anguera et al., 2018).

\section{RESULTADOS}

Os resultados apresentados abaixo pretendem, através de diferentes técnicas estatísticas e da técnica de coordenadas polares, analisar os golos marcados no Mundial 2018. Esta análise pretende ser feita desde a recuperação da bola até ao momento de finalização 


\section{Golos no Campeonato do Mundo na Rússia}

efetiva (golo). Na tabela 3 são apresentados resultados descritivos e a comparação dos golos marcados tendo em conta a fase da competição. A figura 4 mostra-nos um gráfico de barra de erros, para um intervalo de confiança de $95 \%$, para as duas fases da competição, estando plasmado as diferenças estatisticamente significativas dos golos obtidos nas duas fases da competição. As tabelas 4, 5 e as figuras 5 e 6 mostramnos análise das coordenadas polares e o mapa dos vetores para a conduta focal, respetivamente.

Tabela 3. Análise notacional dos golos

\begin{tabular}{|c|c|c|c|c|c|c|c|c|}
\hline \multirow[b]{2}{*}{ Critério } & \multirow[b]{2}{*}{ Categorias } & \multirow{2}{*}{$\begin{array}{c}\text { Fase de } \\
\text { Grupos } \\
\text { Soma } \\
\end{array}$} & \multirow{2}{*}{$\begin{array}{c}\begin{array}{c}\text { Fase de } \\
\text { Eliminação }\end{array} \\
\text { Soma }\end{array}$} & \multirow[b]{2}{*}{ Soma } & \multirow[b]{2}{*}{$M \pm D P$} & \multicolumn{3}{|c|}{ Teste de Fisher } \\
\hline & & & & & & Valor & Sig. & $\begin{array}{l}\text { Coeficiente de } \\
\text { Contingência }\end{array}$ \\
\hline \multirow{10}{*}{ 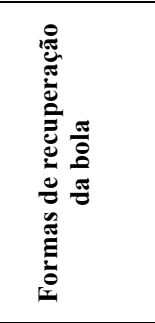 } & GR & 1 & 4 & 5 & $0.11 \pm 0.37$ & 4.27 & 0.07 & 0.29 \\
\hline & INT & 21 & 7 & 28 & $0.61 \pm 0.80$ & 1.33 & 0.76 & 0.17 \\
\hline & DA & 4 & 1 & 5 & $0.11 \pm 0.37$ & 0.70 & 1.00 & 0.09 \\
\hline & DES & 13 & 3 & 16 & $0.35 \pm 0.56$ & 0.88 & 0.86 & 0.16 \\
\hline & BP & 4 & 0 & 4 & $0.09 \pm 0.28$ & - & 0.29 & 0.20 \\
\hline & FALTA & 41 & 14 & 55 & $1.20 \pm 1.14$ & 7.03 & 0.16 & 0.38 \\
\hline & SBLF & 23 & 10 & 33 & $0.72 \pm 1.00$ & 0.43 & 1.00 & 0.05 \\
\hline & SBLL & 14 & 7 & 21 & $0.46 \pm 0.75$ & 1.23 & 0.91 & 0.13 \\
\hline & SBMC & 0 & 0 & 0 & $0.00 \pm 0.00$ & - & - & - \\
\hline & CFP & 1 & 0 & 1 & $0.02 \pm 0.14$ & - & 1.00 & 0.09 \\
\hline \multirow{14}{*}{ 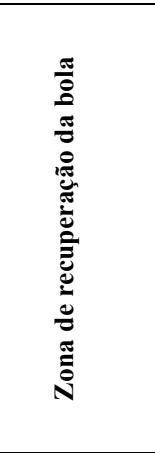 } & ZRDE & 0 & 2 & 2 & $0.04 \pm 0.29$ & - & 0.28 & 0.23 \\
\hline & ZRDC & 4 & 3 & 7 & $0.15 \pm 0.51$ & 3.38 & 0.15 & 0.28 \\
\hline & ZRDD & 2 & 1 & 3 & $0.07 \pm 0.25$ & - & 1.00 & 0.01 \\
\hline & ZRMDE & 8 & 4 & 12 & $0.26 \pm 0.49$ & 1.05 & 0.79 & 0.14 \\
\hline & ZRMDC & 4 & 2 & 6 & $0.13 \pm 0.34$ & - & 1.00 & 0.02 \\
\hline & ZRMDD & 10 & 2 & 12 & $0.26 \pm 0.61$ & 1.05 & 1.00 & 0.14 \\
\hline & ZRMOE & 9 & 3 & 12 & $0.26 \pm 0.49$ & 0.53 & 1.00 & 0.09 \\
\hline & ZRMOC & 11 & 2 & 13 & $0.28 \pm 0.54$ & 0.96 & 0.72 & 0.17 \\
\hline & ZRMOD & 9 & 2 & 11 & $0.24 \pm 0.48$ & 6.76 & $\mathbf{0 . 0 1}^{+}$ & 0.35 \\
\hline & ZROE & 3 & 1 & 4 & $0.09 \pm 0.28$ & - & 1.00 & 0.03 \\
\hline & ZROC & 21 & 5 & 26 & $0.57 \pm 0.68$ & 2.18 & 0.42 & 0.22 \\
\hline & ZROD & 4 & 2 & 6 & $0.13 \pm 0.34$ & - & 1.00 & 0.02 \\
\hline & LL & 14 & 7 & 21 & $0.46 \pm 0.75$ & 1.23 & 0.91 & 0.13 \\
\hline & LF & 23 & 11 & 34 & $0.74 \pm 0.99$ & 0.96 & 0.88 & 0.11 \\
\hline \multirow{13}{*}{ 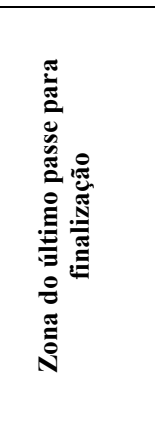 } & ZDE & 0 & 0 & 0 & $0.00 \pm 0.00$ & - & - & - \\
\hline & $\mathrm{ZDC}$ & 0 & 0 & 0 & $0.00 \pm 0.00$ & - & - & - \\
\hline & ZDD & 0 & 0 & 0 & $0.00 \pm 0.00$ & - & - & - \\
\hline & ZMDE & 3 & 0 & 3 & $0.07 \pm 0.25$ & - & 0.54 & 0.17 \\
\hline & ZMDC & 2 & 2 & 4 & $0.09 \pm 0.35$ & 2.56 & 0.36 & 0.25 \\
\hline & ZMDD & 4 & 0 & 4 & $0.09 \pm 0.28$ & 3.06 & 0.29 & 0.20 \\
\hline & ZMOE & 7 & 3 & 10 & $0.22 \pm 0.41$ & - & 1.00 & 0.05 \\
\hline & $\mathrm{ZMOC}$ & 5 & 5 & 10 & $0.22 \pm 0.51$ & 2.21 & 0.30 & 0.19 \\
\hline & ZMOD & 5 & 4 & 9 & $0.20 \pm 0.58$ & 3.59 & 0.37 & 0.27 \\
\hline & $\mathrm{ZOE}$ & 17 & 11 & 28 & $0.61 \pm 0.80$ & 4.94 & 0.13 & 0.32 \\
\hline & $\mathrm{ZOC}$ & 10 & 3 & 13 & $0.28 \pm 0.45$ & - & 0.72 & 0.10 \\
\hline & ZOD & 21 & 7 & 28 & $0.61 \pm 0.71$ & 0.55 & 0.82 & 0.11 \\
\hline & SPF & 46 & 12 & 58 & $1.26 \pm 1.10$ & 8.56 & 0.07 & 0.40 \\
\hline \multirow{11}{*}{ 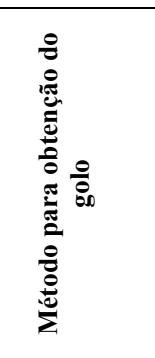 } & $\mathrm{CA}$ & 18 & 5 & 23 & $0.50 \pm 0.81$ & 0.94 & 1.00 & 0.15 \\
\hline & $\mathrm{AR}$ & 13 & 6 & 19 & $0.41 \pm 0.65$ & 2.56 & 0.59 & 0.23 \\
\hline & AP & 37 & 17 & 54 & $1.17 \pm 1.30$ & 5.52 & 0.30 & 0.33 \\
\hline & PEN & 19 & 4 & 23 & $0.50 \pm 0.65$ & 3.42 & 0.17 & 0.26 \\
\hline & LD & 6 & 1 & 7 & $0.15 \pm 0.36$ & - & 0.41 & 0.14 \\
\hline & LI & 11 & 6 & 17 & $0.37 \pm 0.61$ & 0.60 & 0.86 & 0.07 \\
\hline & LLL & 1 & 0 & 1 & $0.02 \pm 0.14$ & - & 1.00 & 0.09 \\
\hline & CL & 15 & 7 & 22 & $0.48 \pm 0.80$ & 0.30 & 1.00 & 0.01 \\
\hline & $\mathrm{CC}$ & 3 & 1 & 4 & $0.09 \pm 0.28$ & - & 1.00 & 0.03 \\
\hline & PB & 0 & 0 & 0 & $0.00 \pm 0.00$ & - & - & - \\
\hline & BS & 0 & 0 & 0 & $0.00 \pm 0.00$ & - & - & - \\
\hline \multirow{6}{*}{ 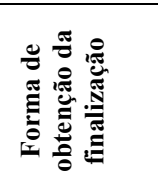 } & PD & 60 & 16 & 76 & $1.65 \pm 1.38$ & 3.21 & 0.72 & 0.27 \\
\hline & $\mathrm{PE}$ & 32 & 13 & 45 & $0.98 \pm 1.23$ & 2.97 & 0.61 & 0.22 \\
\hline & $\mathrm{C}$ & 18 & 15 & 33 & $0.72 \pm 0.83$ & 4.51 & 0.18 & 0.28 \\
\hline & $\mathrm{P}$ & 0 & 0 & 0 & $0.00 \pm 0.00$ & - & - & - \\
\hline & $\mathrm{O}$ & 3 & 1 & 4 & $0.09 \pm 0.28$ & - & 1.00 & 0.03 \\
\hline & $\mathrm{AG}$ & 9 & 2 & 11 & $0.24 \pm 0.43$ & - & 0.46 & 0.14 \\
\hline$\tau$ & $\mathrm{A}$ & 9 & 4 & 13 & $0.28 \pm 0.50$ & 0.75 & 0.80 & 0.11 \\
\hline
\end{tabular}




\section{Santos et al.}

\begin{tabular}{ccccccccc} 
B & 8 & 4 & 12 & $0.26 \pm 0.49$ & 2.49 & 0.38 & 0.24 \\
C & 7 & 0 & 7 & $0.15 \pm 0.36$ & - & 0.08 & 0.27 \\
D & 18 & 7 & 25 & $0.54 \pm 0.86$ & 1.05 & 0.94 & 0.15 \\
E & 16 & 4 & 20 & $0.43 \pm 0.68$ & 1.13 & 1.00 & 0.17 \\
F & $\mathbf{3 1}$ & $\mathbf{1 4}$ & $\mathbf{4 5}$ & $\mathbf{0 . 9 8} \pm \mathbf{0 . 9 5}$ & 6.68 & 0.11 & 0.36 \\
G & 18 & 4 & 22 & $0.48 \pm 0.65$ & 2.74 & 0.29 & 0.24 \\
H & 3 & 1 & 4 & $0.09 \pm 0.28$ & - & 1.00 & 0.03 \\
I & 6 & 7 & 13 & $0.28 \pm 0.54$ & 4.21 & 0.12 & 0.28 \\
J & 6 & 2 & 8 & $0.17 \pm 0.38$ & - & 1.00 & 0.05 \\
\hline
\end{tabular}

Nota. + grau de significância $\mathrm{p}<0,05$

As seleções nacionais marcaram mais golos no mundial em situações de jogo que tiveram início em recuperações de bola resultante de faltas do adversário (FALTA $)(n=55 ; 1.20 \pm 1.14)$. As zonas de recuperação de bola com mais ocorrências foram a linha final (LF) $(n=34 ; 0.74 \pm 0.99)$. De salientar as recuperações de bola a partir do meio-campo ofensivo foram mais efetivas $(n=72)$. O último passe para golo aconteceu, com mais ocorrências, na zona ofensiva direita (ZOD) $(n=28 ; 0.61 \pm 0.71)$ e esquerda (ZOE) $(n=28 ; 0.61 \pm 0.80)$, no entanto é nas situações que não existe passe para finalização (SPF), ou seja, são momentos de finalização que a recuperação de bola (ex.: defesa do guarda-redes, erro de passe do adversário, etc.) é logo seguido de finalização. O golo foi obtido com mais frequência através do método de jogo ataque posicional (AP) $(n=54 ; 1.17 \pm 1.30)$. A forma de finalização com mais ocorrências foi com o pé direito $(\mathrm{PD})(n=76 ; 1.65 \pm 1.38)$ na zona de finalização $\mathrm{F}(n=45 ; 0.98 \pm 0.95)$. As finalizações para golo aconteceram com mais frequência dentro da área de grande penalidade $(n=144)$.

De acordo com a tabela 3 verificamos que somente a zona de recuperação no meio-campo ofensivo direito (ZRMOD) apresenta valores estatisticamente significativos na comparação entre golos, tendo em conta a fase da competição $(p=0,01)$. É ainda nos possível verificar, tendo em conta os coeficientes de contingência, que não existe uma dependência das variáveis estudadas com a fase da competição $(C C<0,40)$.

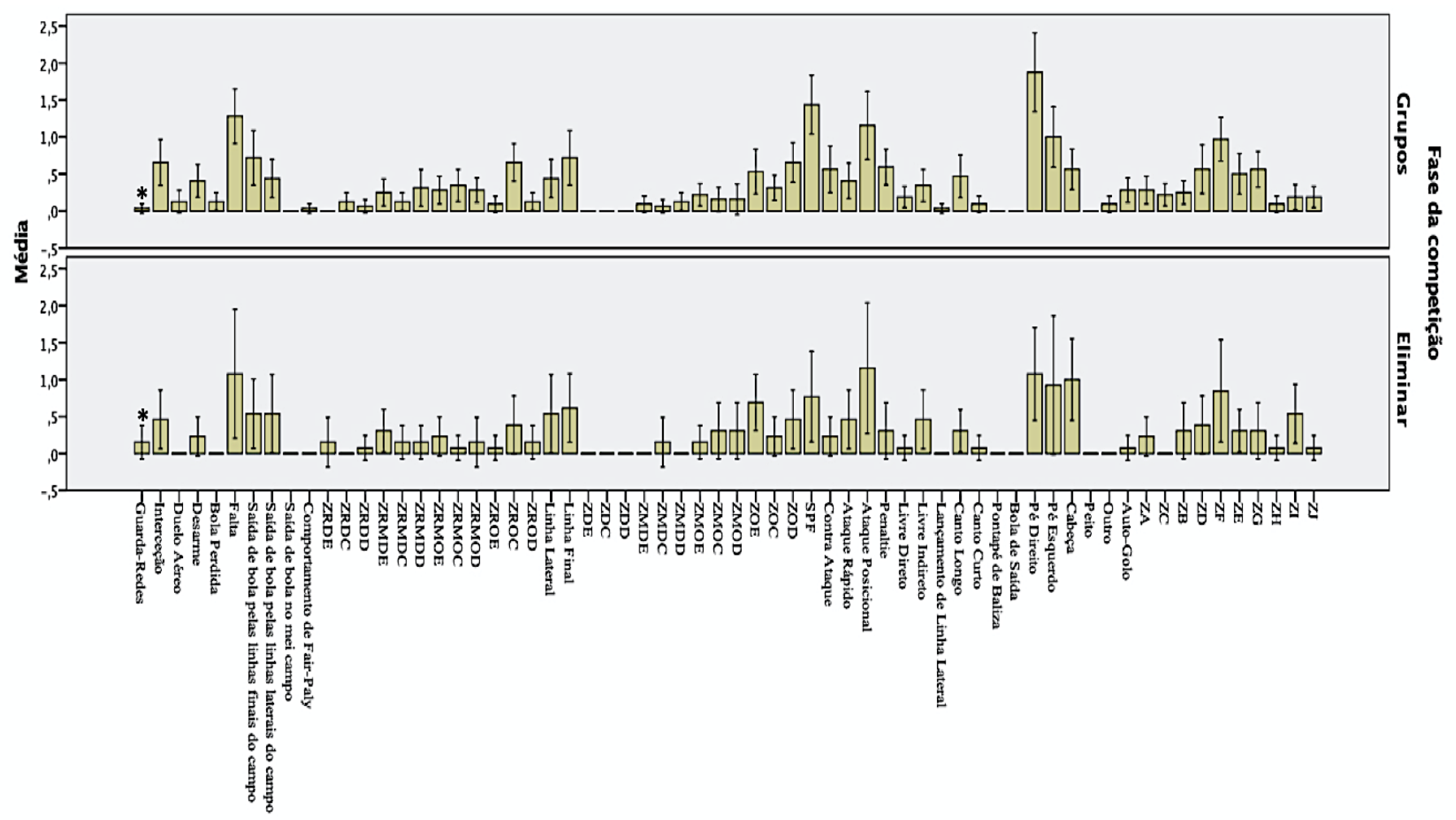

Figura 5. Barra de Erros: Intervalo de Confiança 95\%

${ }^{*}$ Diferenças estatisticamente significativas $(p=0.04)$. Grau significância $p<0.05$ 


\section{Golos no Campeonato do Mundo na Rússia}

$\mathrm{Na}$ figura 4 podemos verificar que existem diferenças estatisticamente significativas entre os golos obtidos na fase de grupos (FG) e na fase a eliminar (FE), na forma de recuperar a bola - guardaredes (FG $-n=1 ; \mathrm{FE}-n=4 ; p=0,04)$. Quanto à recuperação de bola, em ambas as fases, a recuperação de bola acontece fundamentalmente por falta do adversário (FALTA $-\mathrm{FG}-n=41 ; \mathrm{FE}-n=14$ ), saída de bola pela linha final (SBLF $-\mathrm{FG}-n=23$; FE $n=10$ ) e interceção (INT $-\mathrm{FG}-n=21 ; \mathrm{FE}-n=7$ ). As zonas de recuperação de bola com mais ocorrências são a linha final ( $\mathrm{LF}-\mathrm{FG}-n=23 ; \mathrm{FE}-n=11)$, linha lateral (LL $-\mathrm{FG}-n=14 ; \mathrm{FE}-n=7)$ e zona ofensiva central (ZROC $-\mathrm{FG}-n=21$; FG $-n=5$ ). Foram registados mais golos em momentos em que não há passe para finalização (SPF -FG $-n=46 ; \mathrm{FE}-n=12) \mathrm{e}$ com últimos passes na zona ofensiva direita (ZOD $\mathrm{FG}-n=21 ; \mathrm{FE}-n=17)$ e esquerda (ZOE $-\mathrm{FG}-n=7$; $\mathrm{FE}-n=11)$. Os golos são obtidos preferencialmente, fase de grupos, por ataque posicional $(n=37)$, penalidades $(n=19)$ e contra-ataque $(n=18)$, e na fase a eliminar, por ataque posicional $(n=17)$, ataque rápido $(n=6)$ e contra-ataque $(n=5)$. A forma de finalização mais eficaz é com o pé direito ( $\mathrm{FG}-n=60 ; \mathrm{FE}-n=16)$, pé esquerdo (FG $-n=32 ; \mathrm{FE}-n=13)$ e de cabeça (FG $-n=18 ; \mathrm{FE}-n=15)$. Na fase de grupos as finalizações para golo aconteceram na zona $\mathrm{F}(n=31), \mathrm{G}(n=18)$ e $\mathrm{D}(n=18)$ e na fase a eliminar na zona $\mathrm{F}(n=14), \mathrm{D}$ $(n=7)$ e I $(n=7)$
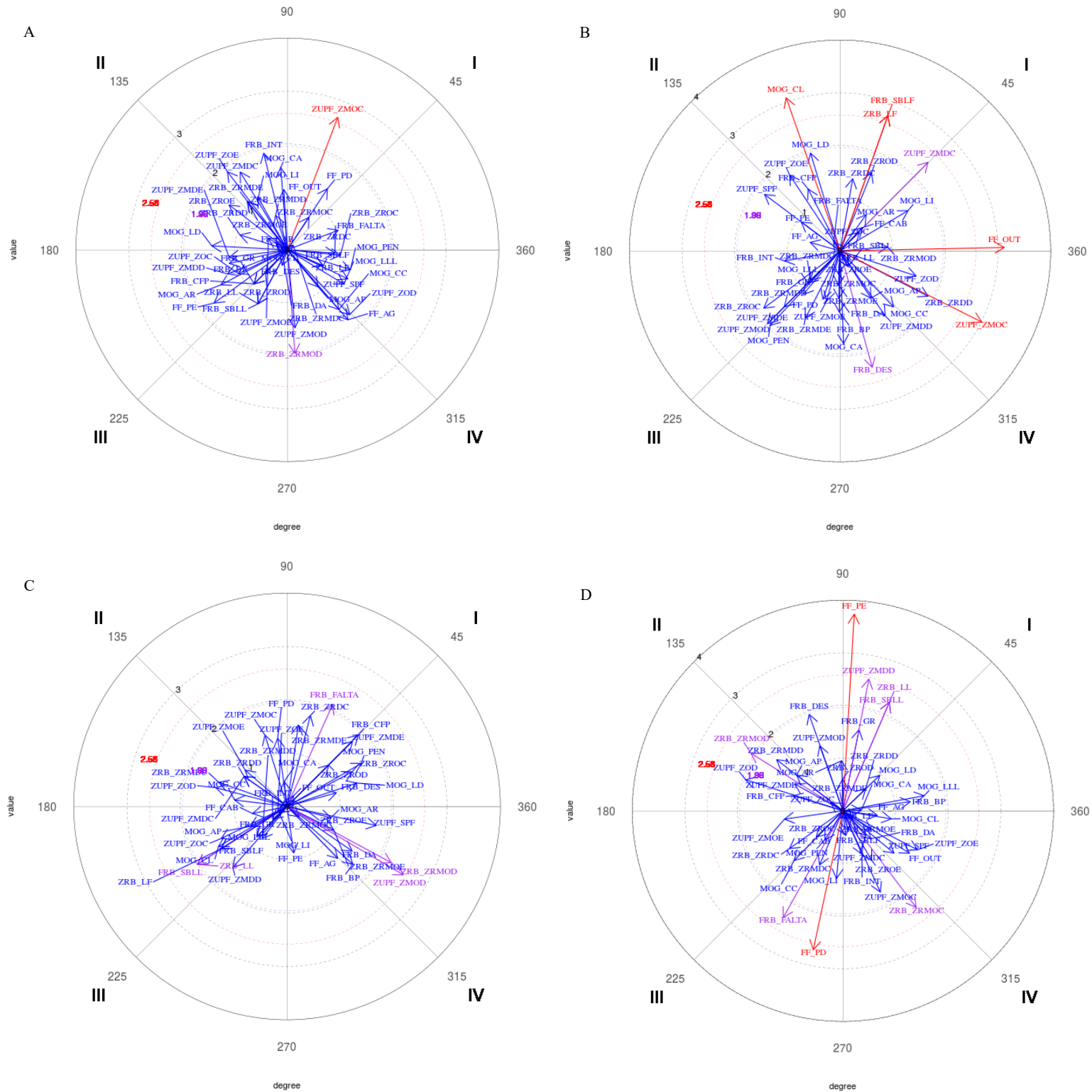
Santos et al.
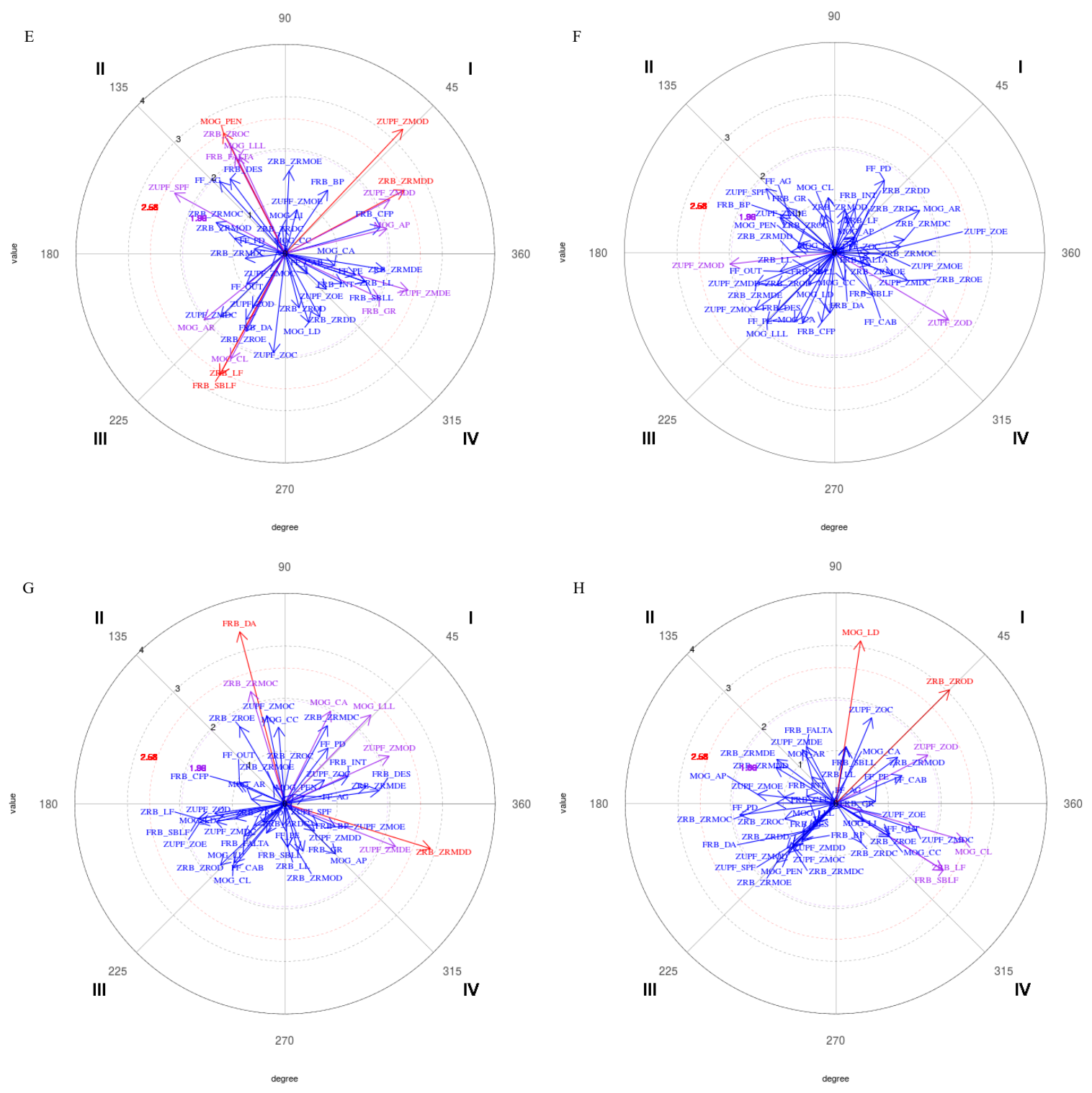


\section{Golos no Campeonato do Mundo na Rússia}
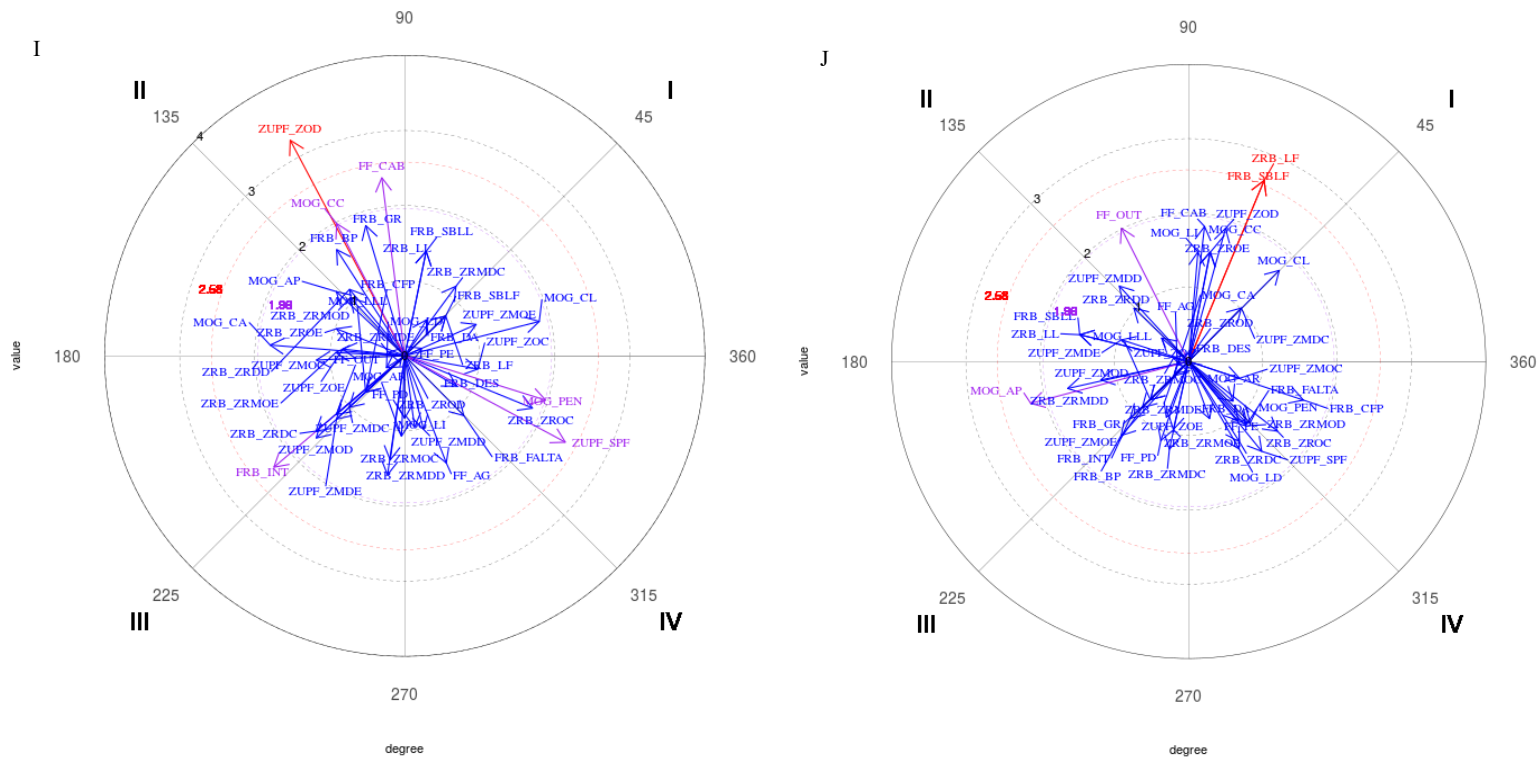

Figura 6. Mapas dos vetores para conduta focal zona de finalização na fase de grupos

Tabela 4. Análise de coordenadas polares entre a conduta focal zonas de finalização e os restantes comportamentos técnico-

\begin{tabular}{|c|c|c|c|c|c|c|}
\hline Conduta Focal & Categoria & Quadrante & Prospetiva & Retrospetiva & Raio & Ângulo \\
\hline \multirow{2}{*}{ A } & ZRMOD & IV & 0.14 & -1.96 & 1.96 & 274.21 \\
\hline & $\mathrm{ZMOC}$ & I & 0.96 & 2.51 & 2.68 & 69.14 \\
\hline \multirow{7}{*}{ B } & DES & IV & 0.61 & -2.2 & 2.29 & 285.48 \\
\hline & SBLF & I & 0.91 & 2.56 & 2.72 & 70.51 \\
\hline & LF & I & 0.91 & 2.56 & 2.72 & 70.51 \\
\hline & ZMDC & I & 1.67 & 1.68 & 2.37 & 45.16 \\
\hline & ZMOC & IV & 2.69 & -1.35 & 3.01 & 333.37 \\
\hline & $\mathrm{CL}$ & II & -1.02 & 2.91 & 3.08 & 109.4 \\
\hline & OUT & I & 3.12 & 0.07 & 3.12 & 1.22 \\
\hline \multirow{5}{*}{$\mathrm{C}$} & FALTA & I & 0.86 & 1.91 & 2.1 & 65.91 \\
\hline & SBLL & III & -1.68 & -1.09 & 2 & 212.87 \\
\hline & ZRMOD & IV & 1.95 & -1.05 & 2.22 & 331.64 \\
\hline & LL & III & -1.68 & -1.09 & 2 & 212.87 \\
\hline & ZMOD & IV & 2.18 & -1.28 & 2.53 & 329.56 \\
\hline \multirow{8}{*}{$\mathrm{D}$} & FALTA & III & -1.13 & -2.03 & 2.32 & 240.81 \\
\hline & SBLL & I & 0.88 & 2.06 & 2.24 & 66.82 \\
\hline & ZRMOC & IV & 1.39 & -1.83 & 2,3 & 307.18 \\
\hline & ZRMOD & II & -1.74 & 1.09 & 2.06 & 147.98 \\
\hline & LL & I & 0.88 & 2.06 & 2.24 & 66.82 \\
\hline & ZMDD & I & 0.49 & 2.5 & 2.55 & 78.82 \\
\hline & PD & III & -0.56 & -2.64 & 2.7 & 257.97 \\
\hline & $\mathrm{PE}$ & I & 0.22 & 3.73 & 3.74 & 86.7 \\
\hline \multirow{15}{*}{$\mathrm{E}$} & GR & IV & 1.8 & -0.85 & 1.99 & 334.71 \\
\hline & FALTA & II & -0.95 & 1.83 & 2.07 & 117.46 \\
\hline & SBLF & III & -1.25 & -2.32 & 2.63 & 241.65 \\
\hline & ZRMDD & I & 2.28 & 1.21 & 2.58 & 28 \\
\hline & ZROC & II & -1.03 & 2.05 & 2.29 & 116.73 \\
\hline & LF & III & -1.25 & -2.32 & 2.63 & 241.65 \\
\hline & ZMDE & IV & 2.35 & -0.7 & 2.45 & 343.41 \\
\hline & ZMDD & I & 2 & 1.03 & 2.25 & 27.29 \\
\hline & ZMOD & I & 2.25 & 2.38 & 3.27 & 46.63 \\
\hline & SPF & II & -2.12 & 1.16 & 2.41 & 151.34 \\
\hline & $\mathrm{AR}$ & III & -1.55 & -1.27 & 2 & 219.25 \\
\hline & AP & I & 1.94 & 0.47 & 2 & 13.74 \\
\hline & PEN & II & -1.18 & 2.31 & 2.59 & 117.1 \\
\hline & LLL & II & -0.87 & 1.86 & 2.05 & 115.11 \\
\hline & $\mathrm{CL}$ & III & -1.04 & -2.01 & 2.26 & 242.68 \\
\hline
\end{tabular}




\section{Santos et al.}

\begin{tabular}{|c|c|c|c|c|c|c|}
\hline & ZMOD & III & -1.99 & -0.22 & 2 & 186.37 \\
\hline $\mathrm{F}$ & ZOD & IV & 2.17 & -1.28 & 2.52 & 329.42 \\
\hline \multirow{7}{*}{ G } & DA & II & -0.86 & 3.7 & 3.38 & 104.73 \\
\hline & ZRMDD & IV & 2.77 & -0.85 & 2.9 & 342.86 \\
\hline & ZRMOC & II & -0.65 & 2.13 & 2.23 & 107 \\
\hline & ZMDE & IV & 2.1 & -0.81 & 2.26 & 338.83 \\
\hline & ZMOD & I & 1.98 & 0.9 & 2.18 & 24.44 \\
\hline & $\mathrm{CA}$ & I & 0.87 & 1.76 & 1.96 & 63.71 \\
\hline & LLL & I & 1.64 & 1.69 & 2.35 & 45.89 \\
\hline \multirow{6}{*}{$\mathrm{H}$} & SBLF & IV & 2.04 & -1.27 & 2.4 & 328.03 \\
\hline & ZROD & I & 2.16 & 2.16 & 3.05 & 44.99 \\
\hline & LF & IV & 2.04 & -1.27 & 2.4 & 328.03 \\
\hline & ZOD & I & 1.74 & 0.91 & 1.97 & 27.73 \\
\hline & LD & I & 0.47 & 3.08 & 3.12 & 81.37 \\
\hline & $\mathrm{CL}$ & IV & 2.44 & -0.71 & 2.54 & 343.73 \\
\hline \multirow{6}{*}{ I } & INT & III & -1.75 & $\begin{array}{l}-1.49 \\
\end{array}$ & 2.29 & 220.37 \\
\hline & ZOD & II & -1.53 & 2.87 & 3.25 & 118.01 \\
\hline & SPF & IV & 2.14 & -1.15 & 2.43 & 331.74 \\
\hline & PEN & IV & 1.88 & -0.59 & 1.97 & 342.52 \\
\hline & $\mathrm{CC}$ & II & -0.91 & 1.76 & 1.98 & 117.28 \\
\hline & $\mathrm{CAB}$ & II & -0.31 & 2.37 & 2.39 & 97.35 \\
\hline \multirow{4}{*}{$\mathrm{J}$} & SBLF & I & 1.02 & 2.43 & 2.64 & 67.36 \\
\hline & LF & I & 1.02 & 2.43 & 2.64 & 67.36 \\
\hline & AP & III & -2.13 & -0.56 & 2.2 & 194.86 \\
\hline & OUT & II & -0.91 & 1.8 & 2.01 & 116.8 \\
\hline
\end{tabular}

$\mathrm{Na}$ figura 7 e tabela 5 mostram-nos os resultados relativos à análise de coordenadas polares, na fase de grupos, tendo como conduta focal as zonas de finalização. No quadrante I, onde existe relações de mútua excitação, facto registado entre a zona de finalização (ZF) A e ZMOC; a ZFB e SBLF, LF e ZMDC; a ZFC e FALTA; ZFD e SBLL, LL e ZMDD; a ZFE e ZRMDD, ZMDD, ZMOD e AP; a ZFG e ZMOD, CA e LLL; a ZFH e ZROD, ZOD e LD e a ZFJ e SBLF e LF. No quadrante II as relações que se estabelecem são excitatórias na perspetiva retrospetiva e inibitória na perspetiva prospetiva, podendo ser verificadas entre a ZFA e CL; a ZFD e ZRMOD; ZFE e FALTA,
ZROC, SPF, PEN e LLL; a ZFG e DA e ZRMOC; a ZFI e $\mathrm{ZOD}, \mathrm{CC}$ e $\mathrm{CAB}$ e a $\mathrm{ZFJ}$ e OUT. No que concerne às relações que se inibem mutuamente (quadrante III), foram registadas entre a ZFC e SBLL e LL; a ZFD e FALTA e PD; a ZFE e SBLF, LF, AR e CL; a ZFF e ZMOD; a ZFI e INT e a ZFJ e AP. No quadrante IV as relações ativam-se na perspetiva prospetiva e inibem-se retrospetivamente, tendo sido verificadas entre a ZFA e ZMOD; a ZFB e DES e ZMOC; a ZFC e ZRMOD e ZMOD; a ZFD e ZRMOC; a ZFE e GR e ZMDE; a ZFF e ZOD; a ZFG e ZRMDD e ZMDE; a ZFH e SBLF, LF e CL e a ZFI e SPF e PEN.
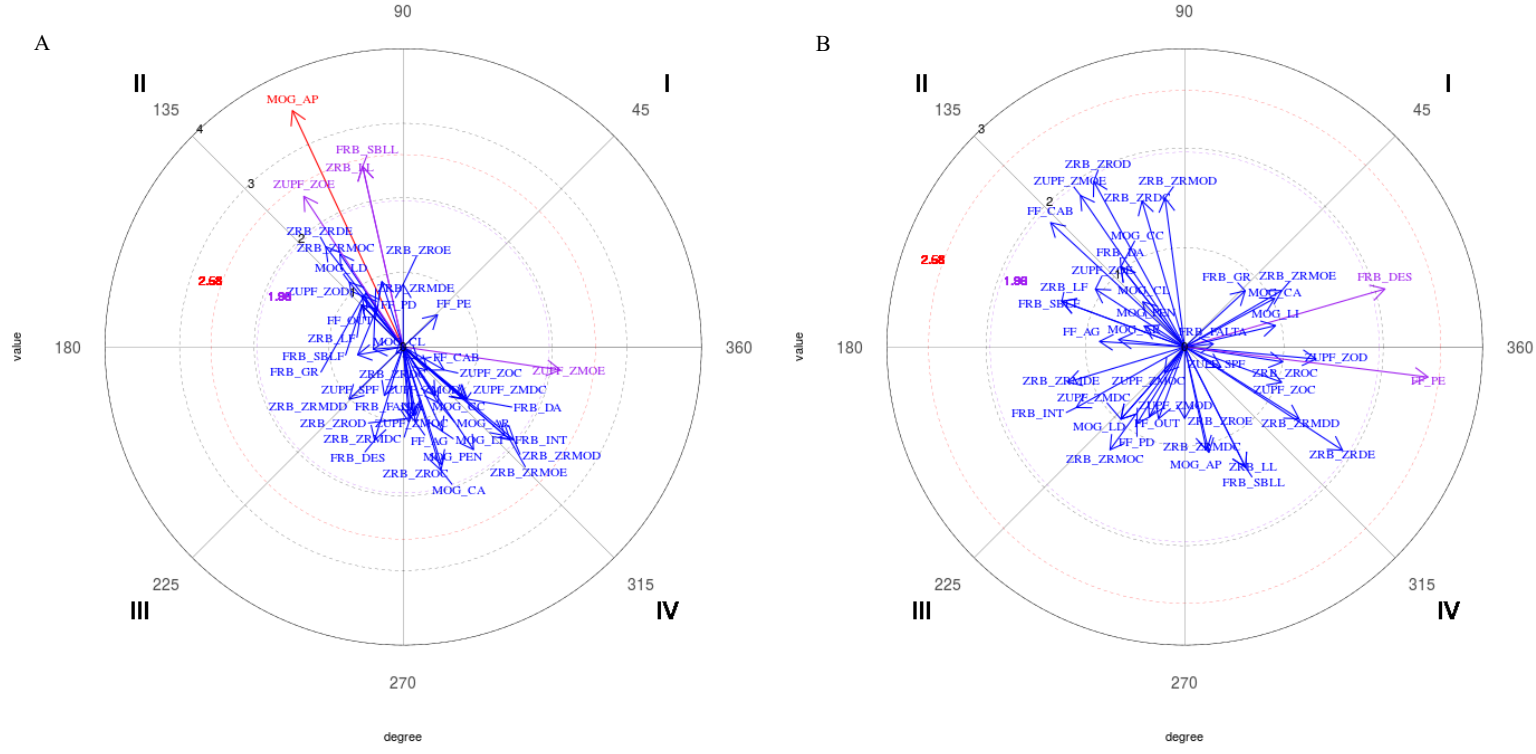


\section{Golos no Campeonato do Mundo na Rússia}
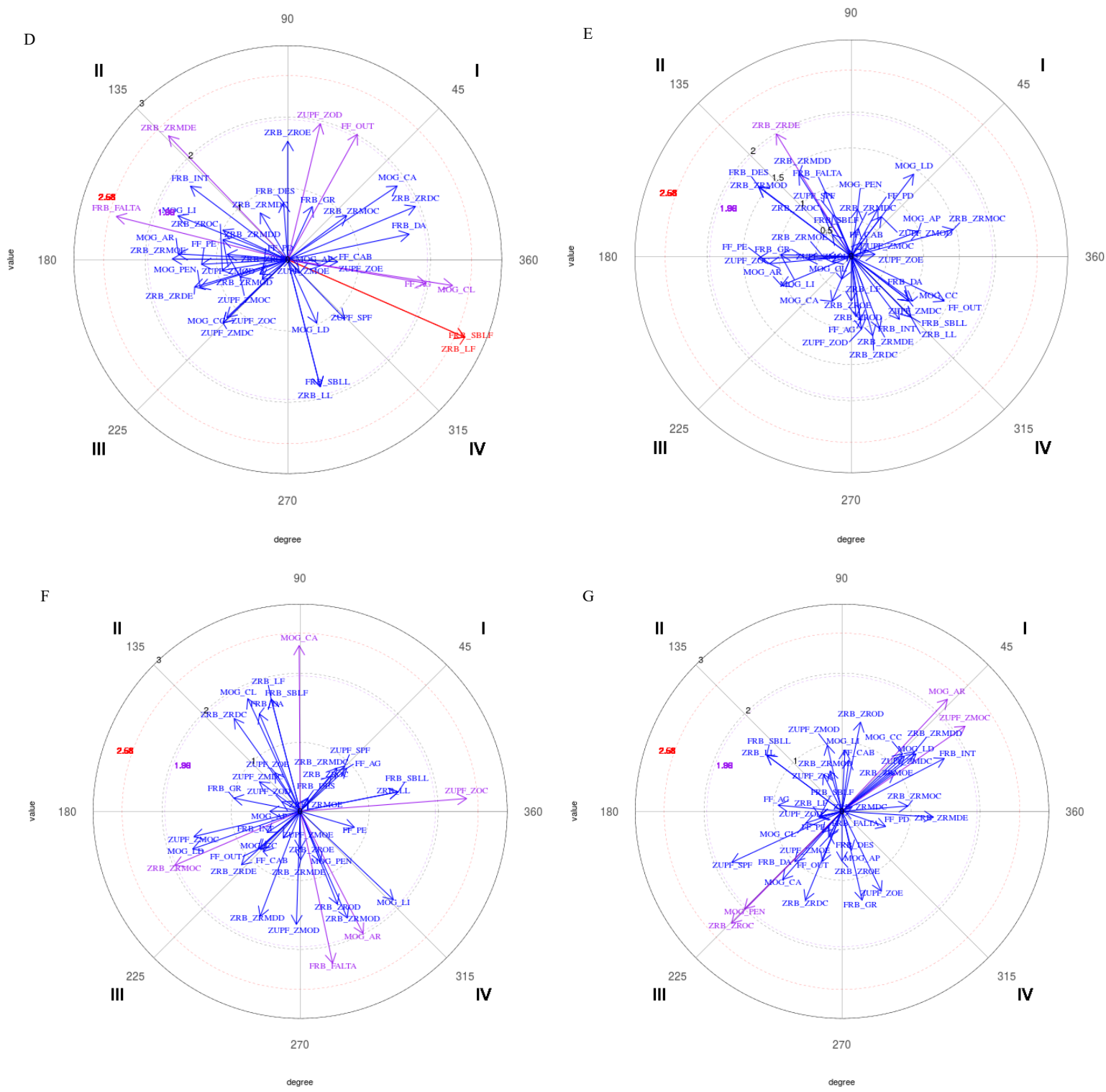


\section{Santos et al.}
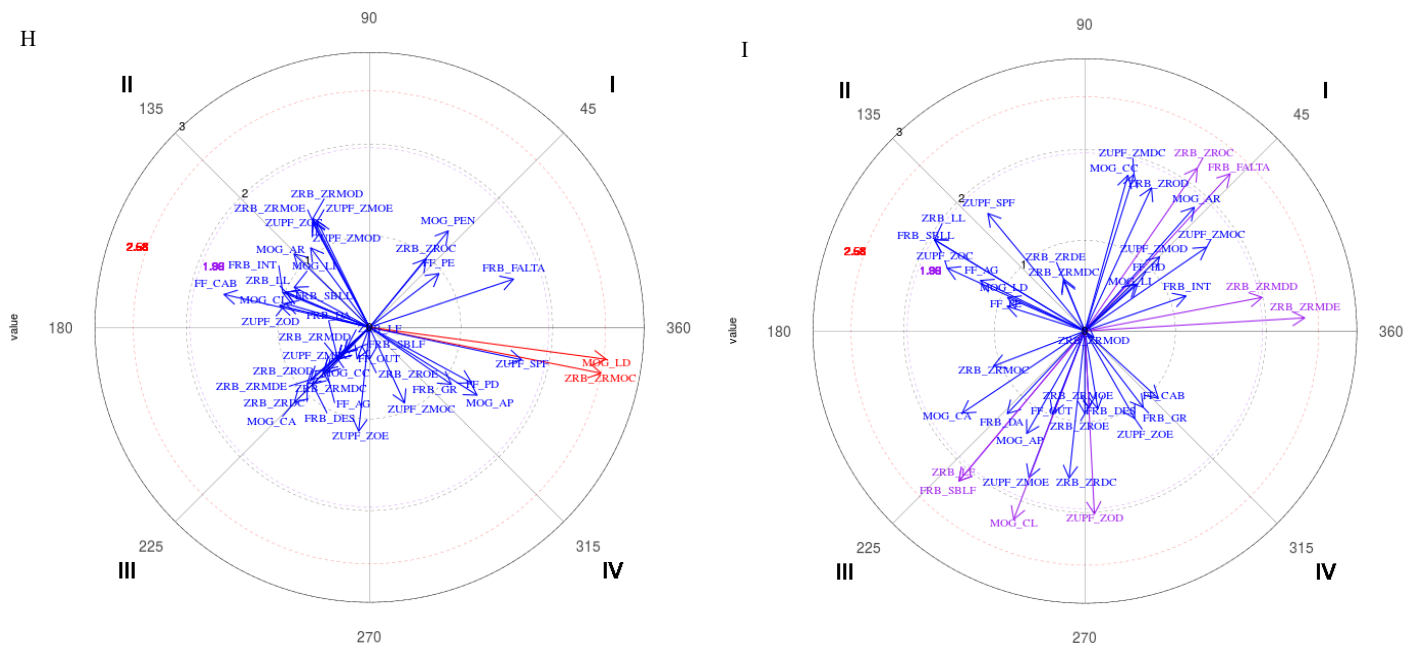

Figura 7. Mapas dos vetores para conduta focal zona de finalização na fase a eliminar

Tabela 5. Análise de coordenadas polares entre a conduta focal zonas de finalização e os restantes comportamentos técnicotáticos na fase a eliminar

\begin{tabular}{|c|c|c|c|c|c|c|}
\hline Conduta Focal & Categoria & Quadrante & Prospetiva & Retrospetiva & Raio & Ângulo \\
\hline \multirow{5}{*}{ A } & SBLL & II & -0.54 & 2.42 & 2.48 & 102.68 \\
\hline & LL & II & -0.54 & 2.42 & 2.48 & 102.68 \\
\hline & ZMOE & IV & 2.1 & -0.31 & 2.13 & 351.65 \\
\hline & $\mathrm{ZOE}$ & II & -1.33 & 2.02 & 2.42 & 123.42 \\
\hline & AP & II & -1.49 & 3.17 & 3.5 & 115.12 \\
\hline \multirow{2}{*}{$\mathrm{B}$} & DES & I & 2.02 & 0.58 & 2.1 & 16.12 \\
\hline & $\mathrm{PE}$ & IV & 2.45 & -0.3 & 2.47 & 352.94 \\
\hline \multirow{7}{*}{$\mathrm{D}$} & FALTA & II & -2.41 & 0.61 & 2.48 & 165.87 \\
\hline & ZRMDE & II & -1.67 & 1.73 & 2.41 & 133.94 \\
\hline & LF & IV & 2.48 & -1.09 & 2.71 & 336.31 \\
\hline & ZOD & I & 0.46 & 1.9 & 1.96 & 76.51 \\
\hline & $\mathrm{CL}$ & IV & 2.31 & -0.36 & 2.34 & 351.07 \\
\hline & OUT & I & 0.97 & 1.75 & 2.01 & 60.98 \\
\hline & $\mathrm{AG}$ & IV & 1.94 & -0.33 & 1.97 & 350.3 \\
\hline $\mathrm{E}$ & ZRDE & II & -1.04 & 1.69 & 1.99 & 121.5 \\
\hline \multirow{4}{*}{$\mathrm{F}$} & FALTA & IV & 0.47 & -2.2 & 2.25 & 282.06 \\
\hline & $\mathrm{ZOC}$ & I & 2.41 & 0.19 & 2.42 & 4.39 \\
\hline & $\mathrm{CA}$ & II & -0.01 & 2.4 & 2.4 & 90.35 \\
\hline & AR & IV & 0.91 & -1.77 & 1.99 & 297.28 \\
\hline \multirow{4}{*}{ G } & ZROC & III & -1.6 & -1.62 & 2.28 & 225.34 \\
\hline & ZMOC & I & 1.78 & 1.23 & 2.17 & 34.62 \\
\hline & AR & I & 1.53 & 1.62 & 2.23 & 46.68 \\
\hline & PEN & III & -1.42 & -1.42 & 2 & 225 \\
\hline \multirow{3}{*}{$\mathrm{H}$} & ZRDE & IV & 3.82 & -0.5 & 3.85 & 352.55 \\
\hline & ZRMOC & IV & 2.53 & -0.5 & 2.58 & 348.82 \\
\hline & LD & IV & 2.59 & -0.35 & 2.61 & 352.32 \\
\hline \multirow{7}{*}{ I } & FALTA & I & 1.6 & 1.73 & 2.36 & 47.22 \\
\hline & SBLF & III & -1.39 & -1.66 & 2.16 & 229.95 \\
\hline & ZRMDE & I & 2.43 & 0.14 & 2.43 & 3.39 \\
\hline & ZRMDD & I & 1.95 & 0.37 & 1.99 & 10.71 \\
\hline & ZROC & I & 1.24 & 1.79 & 2.18 & 55.34 \\
\hline & LF & III & -1.39 & -1.66 & 2.16 & 229.95 \\
\hline & ZOD & IV & 0.11 & -2.02 & 2.02 & 272.98 \\
\hline
\end{tabular}





\section{Santos et al.}

A figura 7 e tabela 5 são relativas à análise das coordenadas referente à fase a eliminar da competição. As relações entre a conduta focal e os restantes comportamentos técnico-táticos, ativam-se mutuamente (quadrante I), de acordo com o podemos ver entre a ZFB e DES; a ZFD e ZOD e OUT; a ZFF e ZOC; a ZFG e ZMOC e AR; a ZFI e ZRMDE, ZRMDD, ZROC e PEN. No quadrante II as relações ativam-se retrospetivamente $\mathrm{e}$ inibem-se prospectivamente, tendo sido verificado entre a ZFA e SBLL, LL, ZOE e AP; a ZFD e FALTA e ZRMDE; a ZFE e ZRDE e a ZFF e CA. No quadrante III estão plasmadas as relações que se inibem mutuamente, facto registado entre a ZFF e ZRMOC; a ZFG e ZROC e PEN; a ZFI e SBLF, LF e CL. Quantos às relações registadas no quadrante IV, ativam-se prospectivamente e inibem-se retrospetivamente, são registadas entre a ZFA e ZMOE; a ZFB e PE; a ZFD e SBLF, LF, CL e AG; a ZFF e FALTA e AR; a ZFH e ZRDE, ZRMOC e LD; a ZFI e ZOD.

\section{DISCUSSÃO}

O objetivo da nossa investigação centra-se na análise do golo, procurando estudá-lo desde o momento da recuperação da bola até ao momento da finalização. Pretendemos ainda verificar, através das coordenadas polares, relações de ativação e inibição entre a conduta focal (zona de finalização) e os restantes comportamentos técnico-táticos.

Nas duas fases da competição analisadas verificámos que as formas de recuperação de bola com mais ocorrências são através da falta sofrida pelo adversário, da saída da bola pelas linhas finais e da interceção. De acordo com a análise de coordenadas polares verificámos várias relações excitatórias ente a conduta focal e o desarme, saída bola pela linha final, falta e saída de bola pela linha lateral. Estudos têm verificado que o desarme e a interceção são as formas mais frequentes de recuperação da bola (Barreira et al., 2014; Hughes \& Lovell, 2019; Liu et al., 2016; Yi et al., 2019). Barreira et al. (2013) refletem no seu estudo, com as seleções nacionais no Mundial de 2010, que as recuperações de bola são influenciadoras da transição defesa-ataque e momento ofensivo. Os autores verificaram que a interceção tende a induzir ataque posicional. De salientar, que foi através do ataque posicional, que as equipas observadas nosso estudo, concretizaram mais golos. Estudos realizados apontam para uma maior eficácia na finalização através dos métodos de jogo ofensivo ataque rápido e o contra-ataque (Espada et al., 2018; GonzálezRodenas et al., 2020; Joaquín Gonzalez-Rodenas et al., 2017; Liu et al., 2015; Tenga et al., 2010). Na análise de coordenadas polares verificámos que a finalização para golo é ativada pelos três métodos de jogo ofensivo.

Barreira et al. (2011) num estudo com as seleções nacionais no Europeu de 2008, também verificaram padrões sequenciais de ações que resultam na finalização, a partir dos lançamentos de linha lateral da linha lateral. Através da análise das coordenadas verificámos, na fase de grupos, que a saída da bola pela linha lateral e a finalização na zona $\mathrm{D}$ ativam-se mutuamente (quadrante I) e na fase a eliminar, a recuperação de bola pela linha lateral é ativadora de finalização na zona A. Também a saída de bola pela linha final, resultando em cantos, tem importância na obtenção do golo, facto evidente na nossa investigação na análise notacional, e nas relações de ativação com a conduta focal finalização, o que vai no sentido que este momento do jogo pode ser relevante para a concretização do golo (González-Ródenas et al., 2019; Leite, 2020).

Relativamente à recuperação da bola pelo guardaredes é também uma evidência no nosso estudo, sendo que este momento é favorável para colocar a bola em áreas de menor pressão do adversário (Barreira et al., 2013). Registámos que esta situação foi mais evidente na fase a eliminar, tendo sido registado diferenças estatisticamente significativas entre as duas fases da competição. No entanto, foi ainda verificada na fase de grupos, que a finalização na zona $\mathrm{E}$ foi ativadora da recuperação da bola na zona $\mathrm{E}$.

No que diz respeito às zonas de recuperação da bola, verificámos um maior número de ocorrências na saída da bola pela linha final, aspeto que já tinha sido realçado relativa à forma de recuperação de bola. Verificou-se que não existem grandes diferenças entre as zonas de recuperação da bola nas zonas média defensiva, zonas médias ofensivas e as zonas ofensivas, tendo sido registadas relações de ativação entre as referidas zonas a finalização em golo. No entanto, é nas zonas médias ofensivas e ofensivas que se fazem mais frequentemente as recuperações da bola que resultaram em golo. Estes dados estão de acordo com os resultados de outros estudos que mostram que as recuperações de bola perto da baliza adversária são mais vantajosas para o golo (Hughes \& Lovell, 2019; Tenga et al., 2010). Devemos salientar, que registámos nas recuperações de bola na zona médio ofensiva 


\section{Golos no Campeonato do Mundo na Rússia}

direita diferenças significativas, tendo em conta a fase da competição. De facto, registámos relações de ativação, na fase de grupos, entre a zona de recuperação média ofensiva direita e as zonas de finalização C (quadrante IV), D (quadrante II) e G (quadrante I). Será importante notar que Barreira et al. (2013) e Espada et al. (2018) verificaram a incidência de recuperações de bola nas zonas defensivas, facto também registado no nosso estudo. As fases finais dos campeonatos de seleções nacionais são provas de curta duração, em que as equipas medem o risco na procura do golo e na defesa da sua baliza. Isto pode ajudar a explicar os resultados da nossa pesquisa, uma vez que as equipas procuram ter um bloco defensivo bastante consistente no processo defensivo (Barreira et al., 2011), promovendo o ataque posicional, método que permite que os jogadores arrisquem no momento certo, realizem uma circulação de bola segura, evitando ser expostos a momentos de transição numa situação de desequilíbrio (Sanjurjo et al., 2015).

De acordo com o acima referido, a importância revelada no nosso estudo para os esquemas táticos (cantos, livres diretos e indiretos e penalidades), podem ser explicadas. Verificámos que na competição de seleções analisada a incidência de golos marcadas por esquemas táticos é considerável, nomeadamente através de cantos longos e grandes penalidades. Nas duas fases da competição foram evidentes, pela análise de coordenadas polares, relações excitatórias entre a finalização resultante em golo e os esquemas táticos canto curto e longo, livre direto e lançamento de linha lateral. Os esquemas táticos revelaram ser situações de jogo relevantes para criar situações de finalização e obter o golo (Acar et al., 2009; González-Ródenas et al., 2019; Kubayi \& Toriola, 2019; Leite, 2020; Li \& Zhao, 2021; Maneiro et al., 2019; Oliva-Millán \& Blanco-Villaeñor, 2011).

Em relação à última zona de passe para a concretização do golo, regista-se com mais frequência nas zonas médias ofensivas e nas zonas ofensivas, tendência verificada em estudos realizados (Amatria et al., 2019b; Clemente et al., 2016; Espada et al., 2018; González-Ródenas et al., 2019). Através das coordenadas polares foi possível verificar que os passes nas zonas médias ofensivas e ofensivas são excitatórios da concretização em golo nas diferentes zonas de finalização. Os resultados do nosso estudo também mostram um número considerável de ocorrências de último passe para a finalização nas zonas médias defensivas, particularmente nos corredores laterais. As equipas após a recuperação da bola, alternam o passe curto e longo e muitas vezes procuram através de um jogo mais direto solicitar os jogadores mais avançados (Malta \& Travassos, 2014). Podemos constatar que passes provenientes das zonas defensivas e zonas médias defensivas, aproveitam os movimentos dos avançados para dentro da área de grande penalidade, ativando a finalização para golo. Em muitas situações de jogo, os golos ocorrem sem um passe final para finalizar, facto que verificamos nos jogos observados. Estas situações ocorrem devido ao posicionamento dos jogadores, beneficiando de erros da equipa adversária no momento de saída de espaços perto da baliza, errando passes, ou mesmo, após uma defesa do guarda-redes. Nestas situações, a recuperação da bola também resulta na finalização para o golo (Santos et al., 2017). Estes momentos são ativadores de finalização na zona $\mathrm{E}$, situação de jogo evidente na fase de grupos da competição.

Quanto à forma de finalizar, verificámos que é de pé direito que os jogadores fazem preferencialmente $o$ golo, seguindo-se o pé esquerdo e cabeça. Estes resultados estão de acordo com o registado em estudos realizados (Acar et al., 2009; Espada et al., 2018; Santos et al., 2017).

De acordo com estudos já realizados, também no nosso estudo foi verificado que é dentro da área de penalidade que a maioria das finalizações resultaram em golo (Acar et al., 2009; Amatria et al., 2019b; Clemente et al., 2016; Espada et al., 2018).

Este estudo fornece alguns indicadores importantes relativamente à forma que as seleções nacionais de alto nível construem situações de finalização e as concretizam em golo. No entanto, temos de considerar como uma limitação do nosso estudo não ter em conta o processo defensivo do adversário, bem como em condições em que se alcança a finalização (inferioridade, igualdade ou superioridade), aspetos que podem favorecer uma melhor caracterização contextual das situações de construção, criação e finalização. Estudos futuros podem desenvolver este sistema de observação de forma a ter em contas as referidas variáveis para caracterização de situações de jogo que resultam em golo. 


\section{Santos et al.}

\section{CONCLUSÕES}

Pretendemos com este estudo verificar como as seleções nacionais, num campeonato do mundo, constroem as suas situações de finalização a partir da recuperação da bola.

Foi possível verificar no nosso estudo que as equipas observadas concretizam muitos dos golos através dos esquemas táticos. Isto foi expresso na análise descritiva, bem na análise feita através de coordenadas polares. Estando de acordo com esta constatação está o facto de muitas ocorrências da forma de recuperação da bola através das faltas cometidas pelos adversários e a zona de recuperação da bola e resultado da saída da bola pela linha final.

Verificámos que a zona de último passe preferencial são o último terço do campo. No entanto, verificámos muitas ocorrências de passes da zona média defensiva para aproveitar as demarcações dos avançados para a finalização. As equipas concretizaram mais golos através do método de jogo ofensivo ataque posicional. Os golos acontecem com maior prevalência dentro da área de penalidade.

\section{APLICACÕES PRÁTICAS}

O nosso estudo analisou os golos marcados no Mundial de Futebol de 2018, através da análise notacional, complementado com o uso de técnicas estatística e utilizando a análise coordenadas polares. Aos treinadores permite o conhecimento de diferentes técnicas para a análise de desempenho de jogadores e equipa, não só do ponto de vista quantitativo, mas também na perspetiva da compreensão dos comportamentos táticos dos jogadores e equipa. Neste sentido, a observação e análise, recorrendo a diferentes técnicas é um importante contributo para melhorar o conhecimento sobre o jogo, bem como para o seu entendimento, sendo um importante contributo na construção de exercícios de treino.

Ao nível do treino, os resultados encontrados reforçam a importância de os treinadores terem em conta na construção do modelo de jogo e exercícios de treino, a importância da recuperação da bola no meio-campo ofensivo, bem como a criação de situações de finalização através de jogo interior (corredor central) e de jogo exterior (corredores laterais). O estudo mostra-nos a grande incidência de golos finalizados dentro da área de grande penalidade, sendo importante o treino reproduzir cenários favoráveis a essa etapa do processo ofensivo. Outra questão que ressalta dos resultados da nossa investigação é o número de golos obtidos através de esquemas táticos (cantos, livres indiretos, livres diretos e grandes penalidades), sendo de todo importante ter presente no planeamento do treino a criação de situações especificas ou dar especial relevo em exercícios competitivos de treino.

\section{REFERÊNCIAS}

1. Acar, M., Yapicioglu, B., Arikan, N., Yalcin, S., Ates, S., \& Ergun, M. (2009). Analysis of goals scored in the 2006 World Cup. The Proceedings of the Sixth World Congress on Science and Football, 235-242.

2. Amatria, M., Maneiro-Dios, R., Pérez-Turpin, J. A., Gomis-Gomis, M. J., Elvira-Aranda, C., \& SuárezLlorca, C. (2019a). Technical-Tactical Analysis of The Players of the Left and Right Wing in Elite Soccer. Journal of Human Kinetics, 70(1), 233-244. doi:10.2478/hukin-2019-0045

3. Amatria, M., Maneiro-Dios, R., \& Anguera-Argilaga, M. T. (2019b). Analysis of the Success of the Spanish National Football Team in the UEFA Euro 2012. Apunts Educació Física $i$ Esports, 137, 85-110. doi:10.5672/apunts.2014-0983.cat.(2019/3).137.07

4. Anguera, M. T., Blanco-Villaseñor, A., Losada, J. L., \& Portell, M. (2018). Pautas para elaborar trabajos que utilizan la metodología observacional. Anuario de Psicología, 48(1), 9-17. doi:10.1016/j.anpsic.2018.02.001

5. Anguera, M. T., \& Hernández-Mendo, A. (2014). Metodología observacional y psicología del deporte: Estado de la cuestión. Revista de Psicología del Deporte., 23, 103-109. doi:10.4321/s157884232015000100002

6. Anguera, M. T., \& Hernández-Mendo, A. (2015). Técnicas de análisis en estudios observacionales en ciencias del deporte Data analysis techniques in observational studies in sport sciences. Cuadernos de Psicología del Deporte, 15(1), 18. doi:10.4321/s157884232015000100002

7. Bakeman, R., \& Quera, V. (2011). Sequential analysis and observational methods for the behavioral sciences. Cambridge University Press.

8. Barreira, D., Garganta, J., \& Anguera, M. T. (2011). In search of nexus between attacking game-patterns, match status and type of ball recovery in European Soccer Championship 2008. Research Methods and Performance Analysis. 5th International Christmas Sport Scientific Conference: Qualitative and Quantitative Research in Sport Science, At Szombathely, Hungary. 


\section{Golos no Campeonato do Mundo na Rússia}

9. Barreira, D., Garganta, J., Guimarães, P., Machado, J., \& Anguera, M. T. (2014). Ball recovery patterns as a performance indicator in elite soccer. Proceedings of the Institution of Mechanical Engineers, Part P: Journal of Sports Engineering and Technology, 228(1), 61-72. doi:10.1177/1754337113493083

10. Barreira, D., Garganta, J., Machado, J. C., \& Anguera, M. T. (2013). Repercussões da recuperação da posse de bola nos padrões de ataque de futebol de elite. Revista Brasileira de Cineantropometria e Desempenho Humano, 16(1), 36-46. doi:10.5007/19800037.2014v16n1p36

11. Blanco-Villaseñor, Á., Castellano, J., HernándezMendo, A., \& Sánchez-López, C. R. (2014). Aplicación de la TG en el deporte para el estudio de la fiabilidad, validez y estimación de la muestra. Revista de Psicología del Deporte., 23(1), 131-137. doi:10.4321/s1578-84232015000100024

12. Brewer, C. J., \& Jones, R. L. (2002). A Five-Stage Process for Establishing Contextually Valid Systematic Observation Instruments: The Case of Rugby Union. The Sport Psychologist, 16(2), 138-159. https://doi.org/10.1123/tsp.16.2.138

13. Castañer, M., Barreira, D., Camerino, O., Anguera, M. T., Canton, A., \& Hileno, R. (2016). Goal Scoring in Soccer: A Polar Coordinate Analysis of Motor Skills Used by Lionel Messi. Frontiers in Psychology, 7. doi:10.3389/fpsyg.2016.00806

14. Castañer, M., Barreira, D., Camerino, O., Anguera, M. T., Fernandes, T., \& Hileno, R. (2017). Mastery in Goal Scoring, T-Pattern Detection, and Polar Coordinate Analysis of Motor Skills Used by Lionel Messi and Cristiano Ronaldo. Frontiers in Psychology, 8, 741. doi:10.3389/fpsyg.2017.00741

15. Castellano, J., \& Hernández-Mendo, A. (2003). El análisis de coordenadas polares para la estimación de relaciones en la interacción motriz en fútbol. Psicothema, 15(4), 569-574. doi: 10.4321/s157884232015000100017

16. Chacón-Moscoso S, Sanduvete-Chaves S, Anguera MT, Losada JL, Portell M and Lozano-Lozano JA (2018) Preliminary Checklist for Reporting Observational Studies in Sports Areas: Content Validity. Frontiers in Psychology; 9:291. doi: 10.3389/fpsyg.2018.00291

17. Clemente, F., Martins, F. M. L., \& Mendes, R. (2016). Analysis of scored and conceded goals by football team throughout a season: A network analysis. Kinesiology, 48, 103-114. https://doi.org/10.26582/k.48.1.5

18. Cochran, W. G. (1954). Some Methods for Strengthening the Common $\chi 2$ Tests. Biometrics, 10(4), 417. doi: $10.2307 / 3001616$

19. Cohen, J. (1960). A Coefficient of Agreement for Nominal Scales. Educational and Psychological Measurement, 20(1), 37-46.

\section{doi:10.1177/001316446002000104}

20. Díaz-Díaz, R., Ramos-Verde, E. J., García-Manso, J. M., Valverde-Esteve, T., \& Arriaza- Ardiles, E. (2018). The use of Polar Coordinates in the analysis of motor interaction in football according to the result. Cuadernos de Psicología del Deporte, 19(1), 60-75. doi:10.6018/cpd.338871

21. Espada, M., Fernandes, C., Martins, C., Leitao, H., Figueiredo, T., \& Santos, F. (2018). Goal characterization after ball recovery in players of both genders of first league soccer teams in Portugal. Human Movement, 2018(5), 73-81. https://doi.org/10.5114/hm.2018.81288

22. Gabin, B., Camerino, O., Anguera, M. T., \& Castañer, M. (2012). Lince: Multiplatform Sport Analysis Software. Procedia - Social and Behavioral Sciences, 46, 4692-4694. doi:10.1016/j.sbspro.2012.06.320

23. González-Rodenas, J., Aranda-Malaves, R., TudelaDesantes, A., Nieto, F., Usó, F., \& Aranda, R. (2020). Playing tactics, contextual variables and offensive effectiveness in English Premier League soccer matches. A multilevel analysis. PLOS ONE, 15(2), e0226978. doi:10.1371/journal.pone.0226978

24. González-Ródenas, J., López-Bondia, I., ArandaMalavés, R., Tudela Desantes, A., Sanz-Ramírez, E., \& Aranda Malaves, R. (2019). Technical, tactical and spatial indicators related to goal scoring in European elite soccer. Journal of Human Sport and Exercise, 15(1). doi:10.14198/jhse.2020.151.17

25. Gonzalez-Rodenas, Joaquín, Lopez-Bondia, I., Calabuig, F., Pérez-Turpin, J. A., \& Aranda, R. (2017). Creation of goal scoring opportunities by means of different types of offensive actions in US major league soccer. Human Movement, 2017(5), 106-116. doi: $10.5114 / \mathrm{hm} .2017 .73616$

26. Gonzalez-Rodenas, Joaquin, Mitrotasios, M., Aranda, R., \& Armatas, V. (2020). Combined effects of tactical, technical and contextual factors on shooting effectiveness in European professional soccer. International Journal of Performance Analysis in Sport, 20(2), 280-293. doi:10.1080/24748668.2020.1743163

27. Gréhaigne, J. F., Mahut, B., \& Fernandez, A. (2001). Qualitative observation tools to analyse soccer. International Journal of Performance Analysis in Sport, 1(1), 52-61. doi:10.1080/24748668.2001.11868248

28. Hernández-Mendo, A., Castellano, J., Camerino, O., Jonsson, G., Blanco-Villaseñor, Á., Lopes, A., \& Teresa Anguera, M. (2014). Programas informáticos de registro, control de calidad del dato, y análisis de datos. Revista de Psicología del Deporte., 23(1), 111-121. doi:10.6018/cpd.423051

29. Hughes, M., \& James, N. (2008). Notational Analysis of Soccer. Kinesiology Research Trends and 


\section{Santos et al.}

Applications, 644-660.

30. Hughes, M., \& Lovell, T. (2019). Transition to attack in elite soccer. Journal of Human Sport and Exercise, 14(1). doi:10.14198/jhse.2019.141.20

31. Jiménez-Salas, J.;Morillo-Baro, J.P.;Reigal, R.E.;Morales-Sánchez, V.;Hernández-Mendo, A. (2020). Análisis de coordenadas polares para el estudio de los sistemas defensivosen balonmano. Cuadernos de Psicología del Deporte,20(1), 103-117. doi:10.6018/cpd.396431

32. Jones, P. D., James, N., and Mellalieu, S. D. (2004). Possession as a performance indicator in soccer. International Journal of Performance Analysis in Sport 4, 98-102. doi: 10.1080/24748668.2004.11868295

33. Kubayi, A., \& Toriola, A. (2019). Trends of Goal Scoring Patterns in Soccer: A Retrospective Analysis of Five Successive FIFA World Cup Tournaments. Journal of Human Kinetics, 69(1), 231-238. doi:10.2478/hukin-2019-0015

34. Leite, W. (2020). The Importance of Set Plays in High Performance Football. Sports Science and Health, 10(1), 5-9. doi:10.7251/JIT2001005L

35. Li, C. \& Zhao, Y. (2021) Comparison of Goal Scoring Patterns in "The Big Five" European Football Leagues. Frontiers in Psychology, 11:619304. doi: 10.3389/fpsyg.2020.619304

36. Liu, H., Gómez, M.-A., Gonçalves, B., \& Sampaio, J. (2016). Technical performance and match-to-match variation in elite football teams. Journal of Sports Sciences, $34(6)$, 509-518. doi:10.1080/02640414.2015.1117121

37. Liu, H., Gomez, M.-Á., Lago-Peñas, C., \& Sampaio, J. (2015). Match statistics related to winning in the group stage of 2014 Brazil FIFA World Cup. Journal of Sports Sciences, 33(12), 1205-1213. doi:10.1080/02640414.2015.1022578

38. Losada, J. L., \& Arnau, J. (2000). Fiabilidad entre observadores con datos categóricos mediante el Anova. Psicothema, 12(Sup2), 335-339.

39. Malta, P., \& Travassos, B. (2014). Caraterização da transição defesa-ataque de uma equipa de Futebol. Motricidade, $10(1)$ 27-37. doi:10.6063/motricidade.10(1).1544

40. Maneiro, R., Amatria, M., Moral, J. E., \& López, G. (2018). Análisis observacional de las relaciones interlíneas de la Selección Española de Fútbol, mediante coordenadas polares. Cuadernos de Psicología del Deporte, 18(2), 18-32. doi:10.4321/s1578-84232015000100018

41. Maneiro R, Casal CA, Ardá A, Losada JL (2019) Application of multivariant decision tree technique in high performance football: The female and male corner kick. PLoS ONE 14(3): e0212549. doi:10.1371/journal.pone.0212549

42. Moreno Cuerva, E. \& Goméz Ruano, M. (2017).
Validación herramienta observacional para el análisis de rachas de lanzamiento en baloncesto. Revista de Psicología del Deporte, 26 (1), 87-93. doi:10.20868/upm.thesis.48327

43. Nevill, A. M., Atkinson, G., Hughes, M. D., \& Cooper, S.-M. (2002). Statistical methods for analysing discrete and categorical data recorded in performance analysis. Journal of Sports Sciences, 20(10), 829-844. doi:10.1080/026404102320675666

44. O'Donoghue, P. (2006). The use of feedback videos in sport. International Journal of Performance Analysis in Sport, 6(2), 1-14. doi:10.1080/24748668.2006.11868368

45. Oliva-Millán, C. \& Blanco-Villaseñor, A. (2011). Codificacíon, registro y análisis de la acción de éxito em fútbol. Cuadernos de Psicología del Deporte, 2011,11(2), 97-103.

46. Portell, M., Anguera, M. T., \& Chacón-Moscoso, S. (2015). Guidelines for reporting evaluations based on observational methodology. Psicothema, 27(3), 283289. doi: $10.7334 /$ psicothema2014.276

47. Portell, M., Anguera, M. T., Hernández-Mendo, A., \& Jonsson, G. (2015). Quantifying biopsychosocial aspects in everyday contexts: An integrative methodological approach from the behavioral sciences. Psychology Research and Behavior Management, 8, 153-160. doi:10.2147/PRBM.S82417

48. Rodríguez-Medina, J., Arias, V., Arias, B., HernándezMendo, A., \& Anguera, M. T. (2019). Polar Coordinate Analysis, from HOISAN to R: A Tutorial Paper. Unpublished manuscript. Retrieved from: ttps://jairodmed.shinyapps.io/HOISAN to_R/

49. Sampaio, J., \& Maçãs, V. (2012). Measuring Tactical Behaviour in Football. International Journal of Sports Medicine, 33(05), 395-401. doi:10.1055/s-00311301320

50. Sanjurjo, C., Losada Lopéz, J. \& Suaréz, T. (2015). Análisis de los factores de rendimiento de las transiciones ofensivas en el fútbol de alto nível. Revista de Psicología del Deporte, 24(1), 103-110. doi:10.18002/10612/910

51. Santos, F., Mendes, B., Maurício, N., Furtado, B., Sousa, P. M., \& Pinheiro, V. (2016). Análise do golo em equipas de elite de futebol na época 2013-2014. REDAF. Revista de Desporto e Actividade Física, 8(1), 11-22.

52. Santos, F., Sarmento, H., Mendes, B., Maurício, N., Furtado, B., Sousa, P. M., \& Pinheiro, V. (2017). Análise complementar do gol no futebol através de análise notacional, análise sequencial e deteção de Tpatterns. Revista Brasileira de Futsal e Futebol, 9(34), 238-249. doi: 10.11606/d.55.2018.tde-28062018113035

53. Sarmento, H., Clemente, F. M., Araújo, D., Davids, K., McRobert, A., \& Figueiredo, A. (2018). What 


\section{Golos no Campeonato do Mundo na Rússia}

Performance Analysts Need to Know About Research Trends in Association Football (2012-2016): A Systematic Review. Sports Medicine, 48(4), 799-836. doi:10.1007/s40279-017-0836-6

54. Tassi, J., Matiz, S. \& Cabezas, M. (2018). El Entrenamiento Psicológico-Integrado en Fútbol a través de Tareas de Entrenamiento. Revista de Psicología Aplicada al Deporte y al Ejercicio Físico, 3(1), 1-15. doi:10.5093/rpadef2018a4

55. Tenga, A., Holme, I., Ronglan, L. T., \& Bahr, R. (2010). Effect of playing tactics on goal scoring in Norwegian professional soccer. Journal of Sports
Sciences,

28(3), doi:10.1080/02640410903502774

56. Yi, Q., Groom, R., Dai, C., Liu, H., \& Gómez Ruano, M. Á. (2019). Differences in Technical Performance of Players From 'The Big Five' European Football Leagues in the UEFA Champions League. Frontiers in Psychology, 10, 2738. doi: 10.3389/fpsyg.2019.02738

57. Vives-Ribó, J., Rabassa, N. (2020). Intervención sobre la comunicación del entrenador para aumentar la confianza de los deportistas. Revista de Psicología Aplicada al Deporte y al Ejercicio Físico, 5(e6), 1-14. doi:10.5093/rpadef2020a1 